NASA/CR-1999-209313

\title{
Modeling of Flow Transition Using an Intermittency Transport Equation
}

Y.B. Suzen and P.G. Huang

University of Kentucky, Lexington, Kentucky

Prepared under Cooperative Agreement NCC3-590

National Aeronautics and

Space Administration

Glenn Research Center 


\section{Acknowledgments}

This work is supported by NASA Glenn Research Center under grant NCC3-590. The project is part of the Low Pressure Turbine Flow Physics program of NASA-Glenn. The contract monitor is Dr. David Ashpis. The authors would like to thank Prof. Peter Bradshaw of Stanford University for his review and his comments.

Available from

NASA Center for Aerospace Information

National Technical Information Service 7121 Standard Drive

Hanover, MD 21076

5285 Port Royal Road

Price Code: A03

Springfield, VA 22100

Price Code: A03 


\title{
Modeling of Flow Transition Using an Intermittency Transport Equation
}

\author{
Y. B. Suzen and P. G. Huang \\ Department Mechanical Engineering \\ Iniversity of hentucky \\ Lexington. Lientucky 40506
}

\begin{abstract}
A new transport equation for intermittency factor is proposed to model transitional flows. The intermittent behavior of the transitional flows is incorporated into the computations by modifying the eddy viscosity. $\mu_{t}$. obtainable from a turbulence model. with the intermitrency factor. $;: \mu_{t}^{*}=i \mu_{t}$. In this paper. Menter s SST model (Menter. 1994) is employed to compute $\mu_{t}$ and other turbulent quantities.

The proposed internittency transport equation can be considered as a blending of two models $\ldots$ Steelant and Dick (1996) and ('ho and ('hung (19992). The former was proposed for near-wall flows and was designed to reproduce the streamwise variation of the intermittency factor in the transition zone following Dhawan and Narasimlac correlation (Dhawan and Varasinhlia, 1958) and the latter was proposed for free shear flows and was used to provide a realistic cross-stream variation of the intermittency profile.

The new morlel was used to predict the T:3 series experinents assembled by Savill (199:3a. 199:3b) including flows with different frest ream turbulence intensit ies and two pressure-gradient cases. For all test cases good agreements between the computed results and the experimental data are observed.
\end{abstract}




\section{Introduction}

To a large extent, flows in low-pressure turbine applications are transitional, and the behavior of the flow transition is strongly affected by the freestream turbulence level and pressure gradients in the turbine blade passage. In order to predict the losses and heat transfer one must be able to predict accurately the boundary-layer development and its interactions with flow transition and separation.

Although a lot of work has been reported on improving the accuracy of the CFD tools in engineering predictions, specially in the turbulence modeling areas, CFD prediction of transitional flows still remains an almost untouched territory, as compared with other areas of CFD developments. A common way to simulate laminar to turbulent transition in computations is to switch on the turbulence model (or turbulent eddy viscosity) at an experimentally pre-determined transition location. This method is ad hoc and ignores the transition physics and the importance of the transitional zone completely. Especially. for flows where the transitional region covers a large portion of the flowfield. as observed in many low-pressure turbine experiments, this practice can lead to severe errors in the solution.

Savill (1993a, 1993b). and Westin and Henkes (1997) have tested a large variety of turbulence models and compared models performances in predicting a few of T:3-series transition flow experiments. They showed that no model could predict both transition location and length for a range of flow conditions and concluded that the existing models were inadequate to predict flow transition.

An alternative to this approach is to use the concept of intermittency to blend the flow from the laminar to the turbulent regions. This approach, although highly empirical. has shown some successes in predicting transition behavior. Dhawan and Narasimha (1958) correlated the experimental data and proposed a generalized intermittency distribution function across flow transition. The correlation was later improved by Gostelow et al. (1994) for flows with pressure gradients subject to a range of freestream turbulence intensities.

Solomon et al. (1995), following the work of Chen and Thyson (1971), developed an improved method to predict transitional flows involving changes in pressure gradients. In this model, the effects of changing streamwise pressure gradient on the breakdown physics and spot spreading rates are taken into account. This is accomplished by varying the spot spreading angle and propagation parameter through the transition zone according to the local pressure gradient parameter.

Steelant and Dick (1996) proposed a transport equation for intermittency, in which the source term of the equation is developed such that the $\gamma$ distribution of Dhawan and Narasimha (1958) across the transition region can be reproduced. Steelant and Dick used their model, coupled with two sets of conditioned Navier-Stokes equations, to predict transitional flows with zero, favorable, and adverse pressure gradients. However. since their technique involved the solution of two sets of strongly coupled equations, the method is not compatible with existing CFD codes. in which only one set of Navier-Stokes equations is involved. Moreover, the model was designed to provide a realistic streamwise $\gamma$ behavior but with no consideration of the variation of $\gamma$ in the cross-stream direction. 
('ho and ('hung (1992) developed a $k-t-\eta$ turbulence model for free shear flows. Their turbulence model explicitly incorporates the int ermit ency effect into the conventional $k-c$ model equations by introducing an additional transport equation for $\gamma$. They applied this model to compute a plane jet. a round jet, a plane far wake, and a plane mixing layer with good agreements. Although this method was not designed to reproduce flow transition it provided a realistic profile of $\gamma$ in the cross-stream direction.

In the current paper. a new transport equation for intermittency factor is proposed. The main motivation in the development of the new model is to combine the best feat ures of the existing transition models in the proposed model. The model can not only reproduce the intermittency distribution of Dhawan and Narasimha in the streamwise direction but also is able to provide a proper variation of $;$ in the cross-st ream direction. Our aim is to propose a model that will predict flow transition under the influences of freestream turbulence and pressure gradients.

A review of a number of transition models used in the current study is given in section 2. In section 3, the new model. combining the best features of the models discussed in section 2. is proposed. Section 4 discusses the numerical issues regarding the implementation of the current method in the existing ('FI) codes. including our choice of the correlations to predict the onset of the transition under the influences of frestream turbulence and pressure gradients. Section 5 shows the comparisons of the new transition model against $T: 3$ series experiments of Savill (19933a. 1993/)). ('oncluding remarks are provided in section 6 .

\section{Transition Models}

\subsection{Dhawan and Narasimha}

Dhawan and Narasimha (1958) correlated a range of experinental data and provided the following expression for streamwise intermittency distribution:

$$
\gamma= \begin{cases}1-\exp \left[-\left(x-x_{t}\right)^{2} n \sigma / l\right] & \left(x \geq x_{t}\right) \\ 0 & \left(x<x_{t}\right)\end{cases}
$$

where $l$ is the freestream velocity: $x$ is the point of transition onset: $n$ is the spot formation rate and $\sigma$ is the spot propagation paraneter.

There are two ways to express equation (1) in dimensionless form: one way. as given by Narasimha (1985), is to express equation (1) in terms of a nondimensional breatidown parameter, $N=n \sigma \theta_{t}^{3} / \nu$, where $\theta_{t}$ is the momentum-thickness at the onset point of transition, $x=x_{t}$, and $\nu$ is the kinematic viscosity: and the other way is in terms of dimensionless spot formation rate. $\hat{n}=m^{2} / l^{3}$, as used by Mayle (1991). Hence. the first part of equation (1) can be written either as:

$$
i=1-\exp \left[-\left(\frac{x-x_{t}}{\theta_{t}}\right)^{2} \frac{\lambda}{R_{t_{\theta_{t}}}}\right]
$$

or

$$
\hat{\imath}=1-\exp \left[-\left(\frac{x-x_{t}}{\theta_{t}}\right)^{2} \hat{n} \sigma R \epsilon_{\theta_{t}}^{2}\right]
$$


Gostelow et al. (1994) conducted measurements of boundary layer transition for six different turbulence levels and a wide range of pressure gradients and correlated the nondimensional breakdown parameter $N$ as:

$$
N= \begin{cases}0.86 \times 10^{-3} \exp \left[2.134 \lambda_{\theta} \ln (T u)-.59 .23 \lambda_{\theta}-0.564 \ln (T u)\right] & \lambda_{\theta} \leq 0 \\ 0.86 \times 10^{-3} \exp [-0.564 \ln (T u)] & \lambda_{\theta}>0\end{cases}
$$

where the pressure gradient parameter, $\lambda_{\theta} \equiv\left(\theta^{2} / \nu\right)(d l / d x)$, and the freestream turbulence intensity, $T u$. are evaluated at the onset point of transition.

In contrast, for zero-pressure-gradient flows, Mayle (1991) correlated the spot production rate using intermittency measurements as;

$$
\hat{n} \sigma=1.25 \times 10^{-11} T u^{7 / 4}
$$

The constant in equation (5) may vary slightly depending on the choice of the data: Mayle also reported a value of $1.5 \times 10^{-11}$ in the same paper. When subject to pressure gradients. Mayle (1991) normalized $\hat{n} \sigma$ by its value at zero-pressure-gradient, $(\hat{n} \sigma)_{Z P G}$, and showed a plot of $\hat{n} \sigma /(\hat{n} \sigma)_{Z P G}$ versus the acceleration parameter, $h=\left(\nu / U^{2}\right)(d t / d x)$ over a wide range of freestream intensity, Tu. Steelant and Dick (1996) proposed the following correlation to fit Mayle's data:

$$
\frac{\hat{n} \sigma}{(\hat{n} \sigma)_{Z P \gamma_{i}}}= \begin{cases}\left(474 T u^{-2.9}\right)^{1-\exp \left(2 \times 10^{6} K\right)}, & K<0 \\ 10^{-3227 K^{-0.5985}}, & K>0\end{cases}
$$

A comparison of equations (2) and (3) indicates that:

$$
N=\hat{n} \sigma R_{t}^{3}
$$

If both Gostelow et al. (1994) and Mayle (1991) used the same data to correlate their dimensionless quantities, $N$ and $\hat{n} \sigma$, respectively, an equation for the Revnolds number at the onset of transition can be identified. For example. for a zero-pressure-gradient case. Gostelow et al.'s (1994) correlation. equation (4), gives:

$$
N=0.86 \times 10^{-3} T u^{-0.564}
$$

Substituting equations ( 8 ) and (5) into equation ( 7 ). yields;

$$
R \epsilon_{\theta_{t}}=410 T u^{-0.7 \pi 1}
$$

Since equation (9) is different from Mayle's correlation (Mayle, 1991);

$$
R_{\epsilon_{t}}=400 T u^{-0.625}
$$

nor does it resemble the well-known Abu-Ghannam and Shaw correlation for zero-pressuregradient flows (Abu-Ghannam and Shaw, 1980):

$$
R_{\theta_{t}}=163+\exp (6.91-T u)
$$

the two correlations, equations (5) and (8), are not identical. We concluded that the choice of the transition correlations using either $N$ or $\hat{n} \sigma$ must be tested in company with a proper choice of the correlations for the onset of transition. 


\subsection{Solomon et al.}

Typically for flows in low-pressure turbines, the onset of transition and the end of transition are in different pressure gradient regions, and to derive transition correlations using only parameters at the onset point of transition may result in an incorrect prediction of the transition length. Following the work of (then and Thyson (1971). Solomon et al. (1995) developed a modified method for calculating intermittency in transitional flows witl changing pressure gradients.

The model of Solomon et al. (1995) accounts for the effects of changing streamwise pressure gradient on the breakdown physics and spreading rate of turbulent spots by allowing the model constants to vary continuously with the local pressure gradient parameter. $\lambda_{f f}$. While the spot generation rate is assumed to depend only on the local conditions at the transition onset point using the relation of (iostelow et al. (1999), equation (t). the spot spreading half-angle $o$ and the spot propagation parameter $\sigma$ are given by:

$$
\begin{aligned}
& \sigma=0.0 .3+\left(0.37 /\left(0.48+3.0 \exp \left(5.2 .9 \lambda_{\theta}\right)\right)\right) \\
& a=4+\left(2 \cdot 2.14 /\left(0.79+2.72 \exp \left(47.63 \lambda_{\theta}\right)\right)\right)
\end{aligned}
$$

The intermittency distribution, retaining the concentrated breakdown hypothesic of Narasimlia. is given by:

$$
\gamma=1-\exp \left[-n \int_{x_{t}}^{x_{t}} \frac{\sigma}{\tan 0} \frac{d x}{l} \int_{x_{t}}^{x_{t}} \operatorname{tanod} d x\right]
$$

where the spot generation rate. $n$. is calculated using.$X$ from equation (4).

Solomon et al. (1995) tested their model for several pressure gradient flows and slowed improved agreement with the experimental data.

\subsection{Steelant and Dick}

Steelant and Dick (1996) developed a transport model for intermittency to be used in conjunction with conditioned Narjer-Stokes ecputions. They derived a model equation starting from the intermittency distribution of Dhawan and Narasimha (1958). Differentiating equation (1) along the streamline direction, s. they arrived at the following transport equation:

$$
\frac{\partial \rho \gamma}{\partial t}+\frac{\partial \rho u\rangle}{\partial x}+\frac{\partial \rho v\rangle}{\partial y}=(1-\lambda) \rho \sqrt{u^{2}+\iota^{2}} 3(. s)
$$

with

$$
f(s)=2 f(s) f^{\prime}(s)
$$

The function $3(s)$ represents the $(n \sigma / C)\left(x-x_{t}\right)$ term in equation (1). The function $f(s)$ is formulated to account for clistributed-breakidown and is given as:

$$
f(s)=\frac{a s^{\prime 4}+b s^{\prime 3}+c s^{\prime 2}+d s^{\prime}+\epsilon}{g s^{n 3}+h}
$$


where the coefficients are;

$$
\begin{gathered}
a=\sqrt{\frac{n \sigma}{U}} \quad b=-0.4906 \quad c=0.204\left(\frac{n \sigma}{U}\right)^{-0.5} \\
d=0.0 \quad \epsilon=0.04444\left(\frac{n \sigma}{U}\right)^{-1.5} \quad g=1.0 \quad h=10 \epsilon
\end{gathered}
$$

Equations (5) and (6) were recommended to evaluate the values of $n \sigma$. In the above equations, $U$ is the freestream velocity at the transition location and the streamline coordinate. $s$. is defined as;

$$
s=\int \frac{u d x+v d y}{\sqrt{u^{2}+v^{2}}}
$$

and $s^{\prime}=s-s_{t}$, where $s_{t}$ is the transition location.

Steelant and Dick (1996) tested their model in conjunction with two proposed sets of conditioned averaged Navier-Stokes equations for zero, adverse, and favorable pressure gradient flows and their results showed that the model performed well for all cases. While the intermittency transport equation reproduces the streamwise intermittency distribution of Dhawan and Narasimha, it gives rise to a fairly uniform $\gamma$ distribution in the cross-stream direction. This is somewhat inconsistent with the experimental observation

of Klelsanoff (1955), in which the variation of intermittency in the cross-stream direction has been proposed empirically as;

$$
\gamma(y)=\frac{1}{2}[1-\operatorname{erf}(\zeta)]
$$

with

$$
\zeta=5\left[\frac{1}{8}\left(\frac{y}{\delta^{*}}\right)-0.78\right]
$$

where $\delta^{*}$ is the displacement thickness.

\subsection{Cho and Chung}

Cho and Chung (1992) developed a $k-t-7$ turbulence model for free shear flows. In their model the intermittency effect is incorporated into the conventional $k-\epsilon$ turbulence model equations explicitly by introducing a transport equation for the intermittency factor $\gamma$. The eddy viscosity is expressed in terms of $h, c$, and $\gamma$. Their intermittency equation was given as:

$$
u_{j} \frac{\partial \gamma}{\partial x_{j}}=D_{\gamma_{i}}+S_{\gamma_{i}}
$$

The diffusion term. $D_{\gamma}$, is represented by:

$$
D_{\gamma_{i}}=\frac{\partial}{\partial x_{j}}\left[(1-\gamma) \frac{\nu_{t}}{\sigma_{g}} \frac{\partial \gamma}{\partial r_{j}}\right]
$$


and the source term, Sis, is;

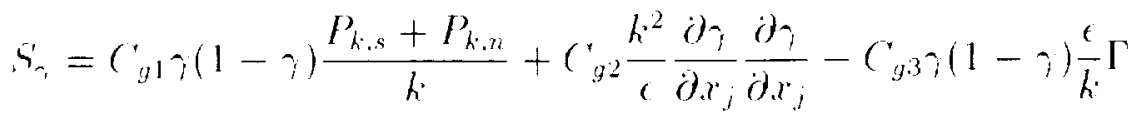

where

$$
\begin{aligned}
& P_{k, s}=-\overline{u_{i} u_{i}} \frac{\partial u_{i}}{\partial x_{,}} \quad(i \neq j) \\
& P_{k, n}=-\overline{u_{i} u_{i}} \frac{\partial u_{i}}{\partial x_{j}} \quad(i=j)
\end{aligned}
$$

and

$$
\Gamma=\frac{k^{5 / 2}}{i^{2}} \frac{u_{i}}{\left(u_{k} u_{k}\right)^{1 / 2}} \frac{\partial u_{i}}{\partial x_{j}} \frac{\partial \gamma_{j}}{\partial x_{j}}
$$

The modeling constants are:

$$
\sigma_{3}=1.0 \quad C_{31}^{\prime}=1.6 \quad C_{32}^{\circ}=0.15 \quad C_{y 3}=0.16
$$

('ho and Chung (1992) tested their model for a plane jet. a round jet. a plane far wake. and a plane mixing layer and showed improved model performance. Althongh the model does indeed produce a very realistic , profile for turbulent free shear flows. it is not designed to predict flow transition to turbulence.

\section{New Transport Model for the Intermittency}

A new transport equation for intermittency is proposed. The main objective of the new model is to reproduce the intermittency distribution of Dhawan and Narasimha in the streamwise direction and at the same time to give rise to a realistic variation of the intermittency in the cross-stream direction. In order to accomplish this. a transport model for intermittency. blending Strelant and Dick's and ('ho and Chung's models. is proposed.

The production term for the new model is a mix of the generation terms of Steclant and Dick and of (ho and ('hung. The first term, $T_{0}$, is from Steeldnt and Dick, aiming to reproduce the intermittency distribution of Dhawan and Narasimha. The formula for' $T_{i}$, is given by:

$$
T_{0}=C_{0} \rho \sqrt{u^{2}+i^{2}} 3(s)
$$

where

$$
3(s)=2 f(s) f^{\prime}(s)
$$


One major aspect in which term $T_{0}$ differs from Steelant and Dick's model is the distributedbreakdown function $f(s)$. Steelant and Dick's model was calibrated for use with conditioned Navier-Stokes equations and when used with the current approach it allows excessively long regions of distributed-breakdown. In order to a obtain shorter distance for distributed-breakdown the function $f(s)$ is modified in the new model.

The function $f(s)$ used in the new model has the same form as Steelant and Dick's model:

$$
f(s)=\frac{a s^{\prime 4}+b s^{\prime 3}+c s^{\prime 2}+d s^{\prime}+c}{g s^{\prime 3}+h}
$$

where the coefficients are;

$$
\begin{aligned}
& a=50 \sqrt{\frac{n \sigma}{\ell}} \quad b=-0.4906 \quad c=0.204\left(\frac{n \sigma}{\ell}\right)^{-0.5} \\
& d=0.0 \quad c=0.04444\left(\frac{n \sigma}{U}\right)^{-1.5} \quad h=10 \epsilon \quad g=50
\end{aligned}
$$

These coefficients are the same as those used in Steelant and Dick's model except for $a$ and g. As will be seen in the comparisons of the models shown in section 5 , the use of equation (17) together with the current intermittency approach, which makes use of intermittency to modify turbulent viscosity directly (see section 4 for details), has a tendency to delay the onset of flow transition. Since the current approach does not use the conditioned Navier-Stokes equations, this adjustment of coefficients, leading to a shorter distance for the distributed-breakdown function $f(s)$, enables a faster response of the mean flow solutions to the intermittent effects.

Two major production terms from Cho and Chung's model are $T_{1}$ and $T_{2}$. These two terms are used $\mathrm{in}$ the form $\left(T_{1}-T_{2}\right)$ in the model. The term $T_{1}$ mimics the production of turbulent kinetic energy, $P_{k}$, and is given by;

$$
T_{1}=C_{1} \gamma \frac{P_{k}}{h}=\frac{C_{i} \gamma}{k} \tau_{i, j} \frac{\partial u_{i}}{\partial r_{j}}
$$

with the shear stresses defined as;

$$
\tau_{i, j}=\mu_{t}\left[\frac{\partial u_{i}}{\partial x_{j}}+\frac{\partial u_{j}}{\partial x_{i}}-\frac{2}{3} \frac{\partial u_{k}}{\partial x_{k}} \delta_{i j}\right]-\frac{2}{3} \rho k \delta_{i j}
$$

The term $T_{2}$ represents the production resulting from the interaction between the mean velocity and the intermittency field and is given by;

$$
T_{2}=C_{2 \gamma \rho} \rho \frac{k^{3 / 2}}{\epsilon} \frac{u_{i}}{\left(u_{k} u_{k}\right)^{1 / 2}} \frac{\partial u_{i}}{\partial x_{j}} \frac{\partial \gamma}{\partial x_{j}}
$$

The production terms $T_{0}$ and $\left(T_{1}-T_{2}\right)$ are blended by using a function $F$ to facilitate a gradual switching from Steelant and Dick's $T_{0}$ to Cho and Chung's $\left(T_{1}-T_{2}\right)$ inside the transition region;

$$
P_{\gamma}=(1-F) T_{0}+F\left(T_{1}-T_{2}\right)
$$


A nondimensional paraneter. $k / S h$, is chosen to construct the blending function $F$. where $h$ is the turbulent kinetic energy and $S$ is the magnitude of the strain rate. This parameter increases rapidly with distance away from the wall inside the transition region. Ising the solution obtained by Dhawan and Varasimha's model coupled with artificial cross-stream profiles provided by Klebanoff's formula, equation (19). one may divide the transition zone into two regions by taking a diagonal cut between the point at the edge of the boundary laver located at the beginning of transition $(\gamma=0)$ to the point at the wall located at the end of transition region $(;=1)$. The relation between $;$ and $\left(k / s_{h}\right)$ along this diagonal line is then approximated by the following correlation:

$$
k / S \nu^{\prime}=200\left(1-\gamma^{0.1}\right)^{0.3}
$$

This line corresponds to the horder between the Steelant and Dick and the ('ho and ('hung models: i.e. below this line. $T_{0}$ is active and above this line $\left(T_{1}-T_{2}\right)$ is active. In order to facilitate a gradual switching from $T_{0}$ to $\left(T_{1}-T_{2}\right)$. the following blending function is proposed:

$$
F=\tanh ^{4}\left[\frac{k / \mathscr{S}_{\nu}}{200\left(1-\gamma^{0.1}\right)^{0.3}}\right]
$$

As can be seen from equation (36). when $k / S i l>>200\left(1-q^{0.1}\right)^{0.3}$ (a position above the cutoff line). $F=1$ and the model switches to (ho and ('hung's model: when $k / h^{\prime} t_{<}<$ $200\left(1-q^{0.1}\right)^{0.3}$ (a position below the cutoff line), $F=0$ and the model becomes Steelant and Dick's model. Ontside the transition zone. the model switch to the ('ho and ('hung:s model except for the very thin region close the wall.

In figure 1. the proposed correlation for the cutoff line equation (35), is compared with data extracted along the cutoff line, defined by drawing a straight line between the point at the edge of the bonndary laver located at the beginning of transition to the point at the wall located at the end of transition region, of the T:A solution obtained using the proposed transition model. As can be seen from the figure. the definition of the cutolf line proposed in equation (35) is satisfactory.

An additional diffusion-related production term is introduced by (ho and chung as:

$$
T_{3}=C_{3 \rho} \frac{k^{2}}{\epsilon} \frac{\partial \gamma}{\partial x_{j}} \frac{\partial \gamma}{\partial x_{j}}
$$

This term is kept active over the entire flowfield. that is. no blending is applied to this terin.

Diffusion of $\gamma$ is represented by the following term:

$$
D_{\gamma_{1}}=\frac{\partial}{\partial x_{j}}\left\{\left[\left(1-\gamma_{\gamma}\right) \sigma_{\gamma_{l}} \mu+\left(1-\gamma_{i}\right) \sigma_{\gamma_{t}} \mu_{t}\right] \frac{\partial \gamma_{\gamma}}{\partial x_{j}}\right\}
$$

The final form of the model is:

$$
\frac{\partial \rho \hat{\imath}_{1}}{\partial t}+\frac{\left.\partial \rho u_{j}\right\rangle}{\partial r_{j}}=\left(1-\gamma_{\gamma}\right)\left[(1-F) T_{0}+F\left(T_{1}-\Gamma_{2}\right)\right]+T_{3}+D_{\hat{\imath}}
$$


or

$$
\begin{aligned}
\frac{\partial \rho \gamma}{\partial t}+\frac{\partial \rho u_{j} \gamma}{\partial x_{j}} & =(1-\gamma)\left[(1-F) C_{0} \rho \sqrt{u^{2}+v^{2}} \beta(s)\right. \\
& \left.+F\left(\frac{C_{1} \gamma}{k} \tau_{i j} \frac{\partial u_{i}}{\partial x_{j}}-C_{2} \rho \rho \frac{k^{3 / 2}}{\epsilon} \frac{u_{i}}{\left(u_{k} u_{k}\right)^{1 / 2}} \frac{\partial u_{i}}{\partial x_{j}} \frac{\partial \gamma}{\partial x_{j}}\right)\right] \\
& +C_{3 \rho} \rho \frac{k^{2}}{\epsilon} \frac{\partial \gamma}{\partial x_{j}} \frac{\partial \gamma}{\partial x_{j}} \\
& +\frac{\partial}{\partial x_{j}}\left(\left((1-\gamma) \gamma \sigma_{\gamma_{l}} \mu+(1-\gamma) \sigma_{\gamma_{t}} \mu_{t}\right) \frac{\partial \gamma}{\partial x_{j}}\right)
\end{aligned}
$$

where the modeling constants are,

$$
\sigma_{\gamma_{1}}=\sigma_{\gamma_{t}}=1.0 \quad C_{0}=1.0 \quad C_{1}=1.6 \quad C_{2}=0.16 \quad C_{3}=0.15
$$

\section{Implementation of the Transition Model}

The intermittency concept can be incorporated into the computations either by using conditioned-average Navier-Stokes equations (Steelant and Dick, 1996; Libby. 1975) or simply by multiplying the eddy viscosity obtained from a turbulence model. $\mu_{t}$. by the intermittency factor. $\gamma$ (Simon and Stephens, 1991). The major difficulty of the former method is the requirement to solve two sets of highly coupled conditioned Navier-Stokes equations. This method is computationally expensive and is not compatible with existing CFD approaches. The latter approach is recommended in the current paper. Simon and Stephens (1991) showed that by combining the two sets of conditioned Navier-Stokes equations and making the assumption that the Reynolds stresses in the nonturbulent part are negligible. the intermittency can be incorporated into the computations by using the eddy viscosity, $\mu_{t}^{*}$. which is obtained by multiplying the eddy viscosity from a turbulence model, $\mu_{t}$, with the intermittency factor. $\gamma$. That is, $\mu_{t}^{*}=\gamma \mu_{t}$ is used in the nean flow equations.

To allow the intermittency to have full control of the transitional behavior. the turbulence model selected to obtain $\mu_{t}$ must produce fully turbulent feature before transition location. Menter's SST model (Menter, 1994) can be shown to produce fully turbulent flow in the leading edge of the boundary layer. It is therefore recommended to be used as a baseline model to compute $\mu_{t}$ and other turbulent quantities in the calculations. Menter's SST model is provided in the Appendix.

The value of $n \sigma$ used in evaluating the constants given by $(30)$ is provided by the Mayle correlation;

$$
\hat{n} \sigma=1.8 \times 10^{-11} T u^{i / 4}
$$

It should be noted that a value of $1.8 \times 10^{-11}$ was used in the current work to give a slightly better fit of Mayle's data. When flows are subject to pressure gradients. the correlation of Steelant and Dick, equation (6), is used.

The current intermittency approach was applied in conjunction with the correlation of Huang and Xiong (1998) for the onset of transition;

$$
\operatorname{Re}_{\theta_{t}}=\left(120+1.50 T u^{-2 / 3}\right) \operatorname{coth}\left[4\left(0.3-h_{t} \times 10^{5}\right)\right]
$$


where $K_{\text {i }}$ is the minimum value of the acceleration parameter in the downstream deceleration region. This correlation was found to provide a slightly better approximation than the correlation of Abu-Ghammam and Shaw (1980) in favorable pressure gradients. as discussed by Huang and Xiong (1998).

The computations are performed with a boundary layer code which solves the mean flow. turbulence model and intermittency equations using second-order finite-volume method. In the computations. 175 grid points. expanding from the wall to the freestream. were used in the cross-st ream direction for all cases. The $y^{+}$values for the first point away from the wall were kept between 0.1 and 0.15 for all cases. The solutions were obtained by using 1000 streamwise steps for all cases. This corresponds to maximum dinemsionless streanwise step sizes, $\Delta x^{+}$. of 49. 68. 47, and 37 for cases T:3.A. T3B, T:36'1, and T:3('2 respectively. These step sizes and cross-stream grid points were found satisfactory by performing a careful grid-independency check. in which the step sizes and grid spacing were both decreased by half: no effect on the solutions was found.

At the inflow, a top-lat velocity profile is prescribed. Inlet turbulent kinetic energy is fixed according to the freestream turbulence levels and the energy dissipation rate is adjusted according to the decay of the freestream turbulence, as will be illustrated in the next section.

\section{$5 \quad$ Results and Discussion}

The new transition model is used to predict the experimental test cases assembled by Savill (1993a, 19933): T:3. T:3B, T:3('1. and T:3(2. These experiments were specially designed to test the ability of turbulence models to predict the effects of freestrean turbulence on the development and subseguent transition of a laminar boundary layer under zcro and rarying pressure gradient conditions. ('ases T3A. T:3B are zero-pressure-gradient flows and T:3('1. T3('2) are cases with continuous change in pressure gradient representing an aft-loaded turbine blade. Comparisons were performed for these cases with the new transition model against the models of I)hawan and Narasimba. Solomon ot al.. and Steelant and Dick.

In all computations, the inlet conditions were calibrated to match the experimental decay of turbulence using the SST model. In each case. the inlet turbulent kinctic energy was fixed by the experimental freestream turbulence level and the matching of the freestrean turbulence clecay provided an estimated value for the dissipation rate of turbulent kinetic energy. $c$. (or the value of $\mu_{t} / \mu$ ) at the inlet. Once the value of $t$ (or $\mu_{t} / \mu$ ) was determined this value was used in subsequent transition computations.

In all cases, the onset of transition was specified according to the correlation of Huang and Xiong (1998). equation (42). The clata of Savill were found to correlate well with equation (42) considering the uncertainties in experinental data; see Huang and Xiong (1998). For T:3A, T3B. T3('1, and T:3C2. the correlation gave the transition locations as $R e_{\theta_{1}}=$ 257, 204. 194. and 297 , respectively, while the experimental kata showed $R_{\theta_{t}}=272.182$. 211 , and 378, respectively. The determination of the experimental onset of transition is rather arbitrary. In our model calculations. we cleliberately used the correlation to predict the onset of transition in order to reduce the dependency on experimental inputs. 
T3A experiment of Savill (199:3a) corresponds to a zero-pressure-gradient flow over a flat plate at $R \epsilon=3.6 \times 10^{5}$ per meter. The freestream turbulence intensity at the leading edge of the flat plate is $3.35 \%$. The decay of freestream turbulence intensity is matched with the experimental data by specifying $\mu_{t} / \mu=7.6$ at the inlet, as shown in Figure 2 .

The computed skin-friction coefficient distributions are compared with the experimental data in Figure 3. Since T3A case is a zero-pressure-gradient case, Dhawan and Narasimha's and Solomon et al.'s models are identical. Steelant and Dick's model showed that the transition was somewhat delayed and the length of transition was slightly wider than the experimental data when compared with the data and the other models. In contrast. the current model predicted the length of the transition region well and also displayed an overshoot of skin-friction coefficient. $C_{f}$, at the end of transition region. It should be noted that the failure of Steelant and Dick's model is because the original distributed-breakdown function, equation (17). was calibrated against the conditioned Navier-Stokes method. To allow for a faster repsonse to flow transition when coupled with the current approach, a modified distributed-breakdown function. equation (29). is recommended.

The comparison of the Reynolds number based on momentum-thickness. Rt ${ }_{\theta}$. for T3A case is shown in Figure 4. As can be seen from this figure. the result of the new model is also in good agreement with the experimental data.

One of the major features of the current model is its ability to reproduce realistic crossstream intermittency profiles. The predicted intermittency profiles at various streamwise stations through the transition zone are shown in Figure 5. The profiles exhibit a peak between $y / \delta^{*}=1$ and $y / \delta^{*}=2$ then drop off toward zero near the edge of the boundary layer. around $y / \delta^{*}=\delta$. These features are consistent with the trends observed in experimental data of Sohn and Reshotko (1991), and Gostelow and Walker (1991).

The current intermittency model does not affect the ability of the turbulence models in the fully developed region, as can be demonstrated in Figure 6. In figures 6(a) and $6(b)$. the velocity profiles obtained using the current intermittency model are compared with those predicted using the SST model without the intermittency modification for $R \epsilon_{\theta}=1,000$ and 5,000. respectively. For $R \epsilon_{\theta}=1000$. the velocity profiles differ slightly near the freestream, indicating the $C_{f}$ value predicted by the current model is slightly larger. This larger value of the skin friction is associated with the overshoot of the skin friction near the end of the transition region, as shown in Figure 3. Further downstream. at $R \epsilon_{\theta}=5000$ the velocity profile obtained from the new model is essentially coincident with the one obtained by using the SST model alone. as shown in figure 6(b). These results clearly show that the new intermittency model does not affect "good" solution behavior of the turbulence model the in the fully developed turbulent region.

The second test case is the T3B case of Savill (199:3a). T3B case is also a zeropressure-gradient flow with a freest ream turbulence intensity of $6 \%$ at the leading edge of the flat plate. The freestream Reynolds number is $6.3 \times 10^{5}$ per meter. In order to match the experimental decay of free stream turbulence intensity. as shown in Figure $\tau, \mu_{t} / \mu$ is specified as 60 at the inlet.

The skin-friction coefficient distributions obtained by the models are compared with the experimental data, as shown in Figure 8 . The transition length predicted by the Dhawan and Narasimha correlation is longer than the data and those predicted by the 
other two models. Steelant and Dick's model showed a delay of transition because of the use of the distributed-breakdown function. equation (17). The new model produces the shortest transition length, and is closest to the experimental data. The discrepancy between the current solution and the experimental data for this case is partly due to the specified transition onset point.

In Figure 9. the momentum-thickness Reynolds number is compared to the experinental data. The solution of the new model is the closest to the experimental data followed by Steelant and Dick's model.

The $\gamma$ profiles from the new model at various streamwise stations through the transition zone are shown in Figure 10. The profiles have the same characteristics as the T3A case. but due to high free stean turbulence intensity. the results showed that the peaks in the profiles are less pronounced for this case and the spread of the intermittency appears to be wider across the transition region.

The next cases are the T36'l and T:36'2 experiments of Savill (1993a). These cases are variable pressure gradient flows representing actual turbine characteristics. Both cases have similar pressure variations. but with different Reynolds numbers and free stream turbulence intensities. The pressure coefficient distributions along the flat plate for these cases are shown in Figure 11. In the figure, the symbols are the experimental data points and the lines are the curve fitting to the data points. These ${ }^{\prime}{ }_{p}$ profiles were input in the boundary layer code to perform the calculations.

T:3('1 case has an inlet Reynolds number of $4.1 \times 10^{5}$ per meter. Turbulence intensity at the leading edge of the plate is $7.78 \%$ and a value of $\mu_{t} / \mu=30$ is used at the inled in order to match the decay of free stream turbulence intensity as shown in Figure 12 .

A comparison of skin-friction is shown in Figure 13 for T3Cl case. All models except the current model overpredicted the transition length. The solutions of Dhawan and Narasimba model and of 1 le model of Solomon et al. are slightly different with the latter predicting a longer 1 ransition length. Again. Steelant and Dick s model showed a delay of the transition due to a slower distributed-breakclown function, equation (17). being used. Overall. the new model showed a fairly good comparison of the transition hehavion.

The $R_{\theta}$ distribution along the flat plate is compared to experimental data in Figure 1.4 for the T:30'l case. In general, the new model reproduced the development of the boundary. laver better than all other models.

The intermittency factor profiles from the new model at various streamwise stations through the transition zone are shown in Figure 15 for the T:36'l case. The characteristics of the profiles are similar to the T:3B cases with mild peaks showing around $y / \delta^{*}=1.6$ and the profiles decaying to zero around $y / \delta^{*}=10$, near the edge of the boundary layer.

T3(2.2 case has an inlet Reynolds number of $3.5 \times 10^{5}$ per meter and a free stream turbulence intensity of $2.8 \%$ at the leading. The decay of free stream turbulence intensity was matched with the the experincental clata by specifying $\mu_{t} / \mu=5$ at the inlet. as shown in Figure 16.

The predicted shin-friction coefficient distributions are compared with experimental data in Figure 17. Both the models of Dhawan and Narasimha and of Solomon et al. give rise to a too early transition behavior and also a longer transition length. On the other hand. Steelant and IJick's model did not predict the onset of transition upto the end of computational domain. Again this is caused by the original distributed-breakdown 
function being used, as discussed earlier. Overall, the current model shows a fairly good comparison with the data.

The Reynolds number based on momentum-thickness along the flat plate is compared to experimental data in Figure 18. Overall, the new model reproduces the development of boundary layer fairly well.

The intermittency factor $\gamma$ profiles at various stations through the transition zone are shown in Figure 19 for the T3C2 case. It should be noted that $\mathrm{T} 3 \mathrm{C} 2 \mathrm{2}$ has a lower freestream turbulence intensity than T3C1. As a results. the profiles show more pronounced peaks between $y / \delta^{*}=1$ and $y / \delta^{*}=2$ and the spread of the intermittency is less wider across the transition zone. These features are similar to the comparison of the zero-pressure-gradient. cases, T3A and T3B.

\section{Concluding Remarks}

A new transport equation for intermittency is developed for modeling transitional flows including influences of free stream turbulence and pressure gradients. The model can be considered as a mix of two existing models, aiming to reproduce the intermittency distribution of Dhawan and Narasimha (1958) in the streamwise direction and to produce a realistic variation of intermittency in the cross-stream direction.

The new model was tested for botll zero-pressure-gradient and variable pressure gradient flows with different freestream turbulence intensities. The new model was used in conjunction with Menter's SST model to predict the T:A. T3B. T3C'1. and T:3C2 experiments of Savill (1993a. 1993b). Comparisons of $C_{f}$ distributions the development of the boundary layer, and intermittency profiles were made for all cases. The new nodel showed a good transition behavior for all cases. 


\section{Appendix - Menter's SST Model}

Menter's SST model (Menter, 1994) is based on a mix of two-equation $k-w$ and $k-c$ turbulence models using a blending function $F_{1}$. The model can be written as:

k-equation:

$$
\frac{\partial \rho k}{\partial t}+\frac{\partial \rho u_{j} k}{\partial x_{j}}=P_{k}-0.09 \rho \omega k+\frac{\partial}{\partial x_{j}}\left(\left(\mu+\sigma_{k} \mu_{t}\right) \frac{\partial k_{i}}{\partial x_{j}}\right)
$$

$\omega$-equation;

$$
\begin{aligned}
\frac{\partial \rho \omega^{\prime}}{\partial t}+\frac{\partial \rho u_{j} \omega}{\partial x_{t}}=\frac{c}{\nu_{t}} P_{k} & -3 \rho \omega^{2}+\frac{\partial}{\partial x_{j}}\left(\left(\mu+\sigma_{\omega} \mu_{t}\right) \frac{\partial \omega^{\prime}}{\partial x_{j}}\right) \\
& +2 \rho\left(1-F_{1}\right) \sigma_{\omega \cdot 2} \frac{1}{\omega} \frac{\partial h_{i}}{\partial x_{j}} \frac{\partial \omega_{j}}{\partial x_{t}}
\end{aligned}
$$

The constants. $c, f, \sigma_{k}$ and $\sigma_{\omega}$. are given by the following general expression:

$$
o=F_{1} \rho_{1}+\left(1-F_{1}\right) o_{2}
$$

where o represents any one of these constants: or represents any constant in the $k-\omega$ model: $b_{2}$ represents the corresponding constant in the $k-e$ model. These constants are defined as:

Set $1(k-\mu):$

$$
\sigma_{k, 1}=0.85 \quad \sigma_{\omega 1}=0.5 \quad \beta_{1}=0.075 \quad c_{1}=0.5 .53
$$

$\operatorname{Sot} 2(k-t)$

$$
\sigma_{k^{2}}=1.0 \quad \sigma_{\omega^{\prime 2}}=0.856 \quad 3_{2}=0.0828 \quad c_{2}=0.44
$$

The production term is given as:

$$
P_{k}=\tau_{i j} \frac{\partial u_{i}}{\partial x_{j}}
$$

where

$$
\tau_{i, j}=\mu_{t}\left[\frac{\partial u_{i}}{\partial x_{j}}+\frac{\partial u_{j}}{\partial x_{i}}-\frac{2}{3} \frac{\partial u_{k}}{\partial x_{k}} \delta_{i j}\right]-\frac{2}{3} \rho k \delta_{i j}
$$

The blending function $F_{1}$ is defined as:

$$
F_{1}=\tanh \left(\arg _{1}^{4}\right)
$$


with

$$
\arg _{1}=\min \left\{\max \left[\frac{\sqrt{k}}{0.09 \omega d} ; \frac{500 \nu}{d^{2} \omega}\right]: \frac{4 \rho \sigma_{\omega 2} k}{C D_{k \omega} d^{2}}\right\}
$$

where $d$ is the distance to the closest wall and $C D_{k w}$ is the positive portion of the crossdiffusion term in Equation (A-2).

$$
C D_{k \omega}=\max \left[2 \rho \sigma_{\omega 2} \frac{1}{\omega} \frac{\partial k}{\partial x_{j}} \frac{\partial \omega}{\partial x_{j}}: 10^{-20}\right]
$$

The kinematic eddy viscosity is defined as;

$$
\nu_{t}=\frac{a_{1} k}{\max \left(a_{1} \omega^{\prime} ; \Omega F_{2}\right)}
$$

where $\Omega$ is the magnitude of vorticity and $a_{1}=0.31$. The function $F_{2}$ is given by:

$$
F_{2}=\tanh \left(\arg _{2}^{2}\right)
$$

with

$$
\arg _{2}=\max \left[\frac{2 \sqrt{k}}{0.09 \omega \cdot d} ; \frac{500 \nu}{d^{2} \omega}\right]
$$




\section{References}

[1] Abu-Ghammam, B.J. and Shaw, R.. 1940. "Natural Transition of Boundary Layers-The Effects of Turbulence, Pressure Gradient. and Flow History." Journal of Mechaniral Engineering Sécience. Vol. 22, No. 5. pp. 21:3-22x.

[2] Chen. K. K. and Thyson. N. A. 1971. "Extension of Emmons' Spot Theory to Flows on Blunt Bodies," AIAA Journal. Vol. 9. No. 5. pp. $821-825$.

[3] Cho, J.R. and C'lung. M.K., 1992, "A li- - - , Equation Turbulence Model," Journal of Fluid Merhanics. Vol. 237. pp. 301-322.

[4] Dhawan, S. and Narasimla, R., 1958. "Some Properties of Boundary Layer During the' Transition from Laminar to Turbulent Flow Notion." Journal of Fluid Mcrhanirs. Vol. 3. pp. $11 x-436$.

[5] Ciostelow, J.P.. Blunden, A.R., and Walker, (i.... 1994. "Effects of Free-Stream Turbulence and Adverse Pressure (iradients on Boundary Layer Transition." ASME Journal of Tiurbomarhinery. Vol. 116. pp. 392-404.

[6] Costelow. J. P. and Walker, C. J.. 1991. "Similarity Behavior in Transitional Boundary Layers Over a Range of Adverse Pressure Ciradients and Turbulence Levels." Jourmal of Turbomachincry. Vol. 113. pp. 617-625.

[7] Huang. P.(i. and Xiong. (i., 1998, .'Transition and Turbulence Modeling of Low Pressure Turbine Flows." AIA Paper AIA -98-0339, Reno. NV.

[s] Klebanoff, P.S. 195.). "Characteristics of Turbulence in a Boundary Layer with Zero Pressure Ciradient." NAC'A Report No. 1247.

[9] Libby, P.A. 1975. "On the Prediction of Intermittent Turbulent Flows." Journal of Fluid Mechanics. Vol. 6x. Part 2. pp. 273-295.

[10] Mayle, R.F.. 1991. "The Role of Laminar-Turbulent Transition in Cias Turbine Engines." Journal of Turbomachinery. Vol. 113. pp. 509 53.

[11] Menter. F. R.. 1991. “Two-Equation Eddy-Viscosity Turbulence Models for Engineering Applications." ALA.4 Joumal, Vol. 32. No. R. August. pp. 1598-1605.

[12] Narasimha, R., 1985, *The Laminar-Turbulent Transition Zone in the Boundary Layer." Progresse in Acrospene Serence. Vol. 22. pp. $29-80$.

[13] Savill. A.M., 1993a. "Some Recent Progress in The Turbulence Modeling of Bry-pass Transition," Near-llall Turbulent Flours, edited by R.M.C. So. ('. (i. Speziale and B.E. Launder, Elsevier Science Publishers B.V.. pp. \$29 $8.1 \%$.

[14] Savill. A.M., 1993b. "Further Progress in The Turbulence Modeling of By-pass Transition," Engine ring Turbulence Modfling and Experiments 2. edited by. W. Rodi and F. Martelli, Elsevier Science Publishers B. V.. pp. 58:3-592. 
[15] Simon. F.F. and Stephens, (.A., 1991. "Modeling of the Heat Transfer in Bypass Transitional Boundary-Layer Flows," NASA Technical Paper 3170.

[16] Soln, Ki-Hyeon and Reshotko, Eli, 1991, "Experimental Study of Boundary Layer Transition With Elevated Freestream Turbulence on a Heated Flat Plate," NASA CR-187068.

[17] Solomon, W.J., Walker, G.J., and Gostelow, J.P., 1995, "Transition Length Prediction for Flows with Rapidly Changing Pressure Gradients", ASME Paper ASME-95-GT-241. International Gas Turbine and Aeroengine Congress \& Exposition, Houston, Texas, June $5-8$.

[18] Steelant. J. and Dick, E. 1996. "Modelling of Bypass Transition with Conditioned NavierStokes Equations Coupled to an Intermittency Transport Equation," International Journal for Numerical Methods in Fluids, Vol. 23, pp. 193-220.

[19] Westin, K. J. A. and Henkes, R. A. W. M., 1997. "Application of Turbulence Models to Bypass Transition." Journal of Fluids Enginfering. Vol.119, pp. 859 866. 


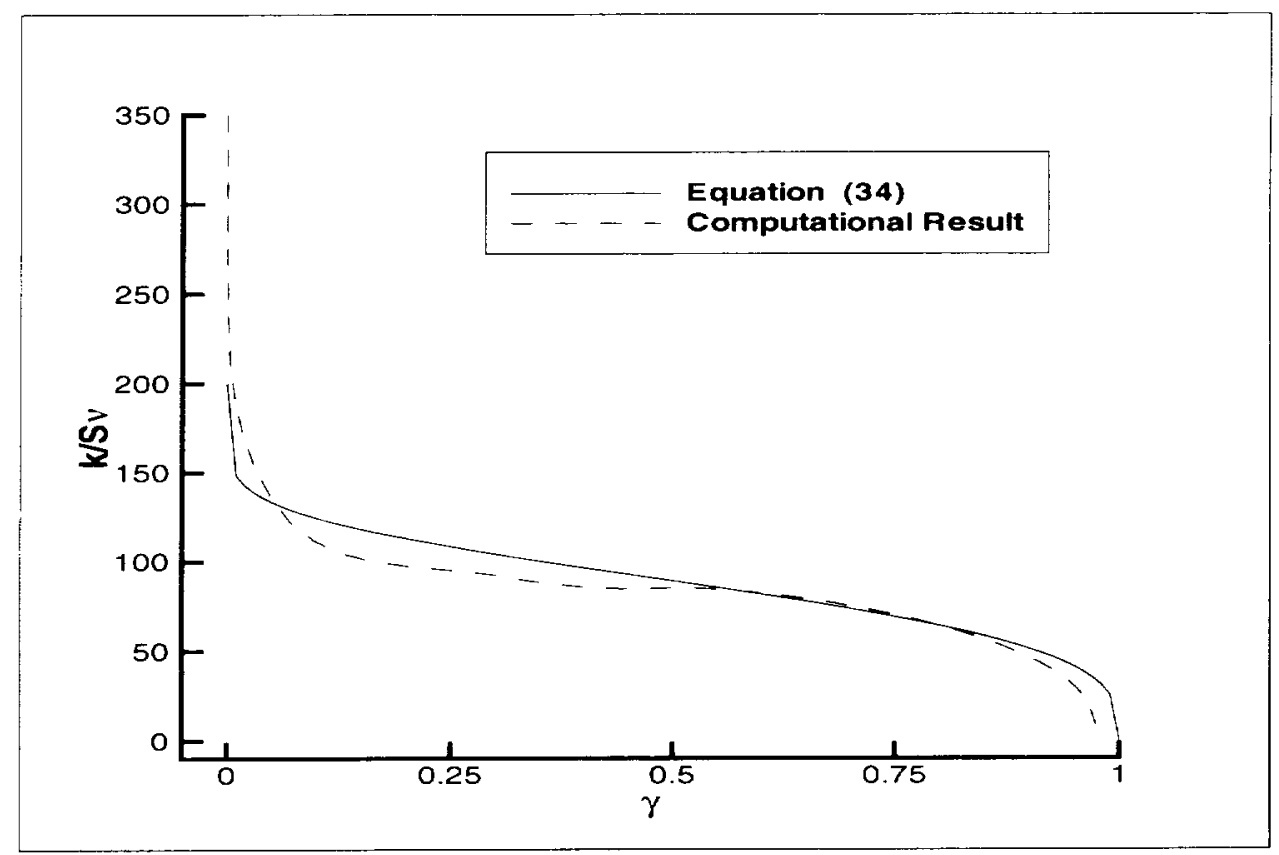

Figure 1: Blending Parameter.

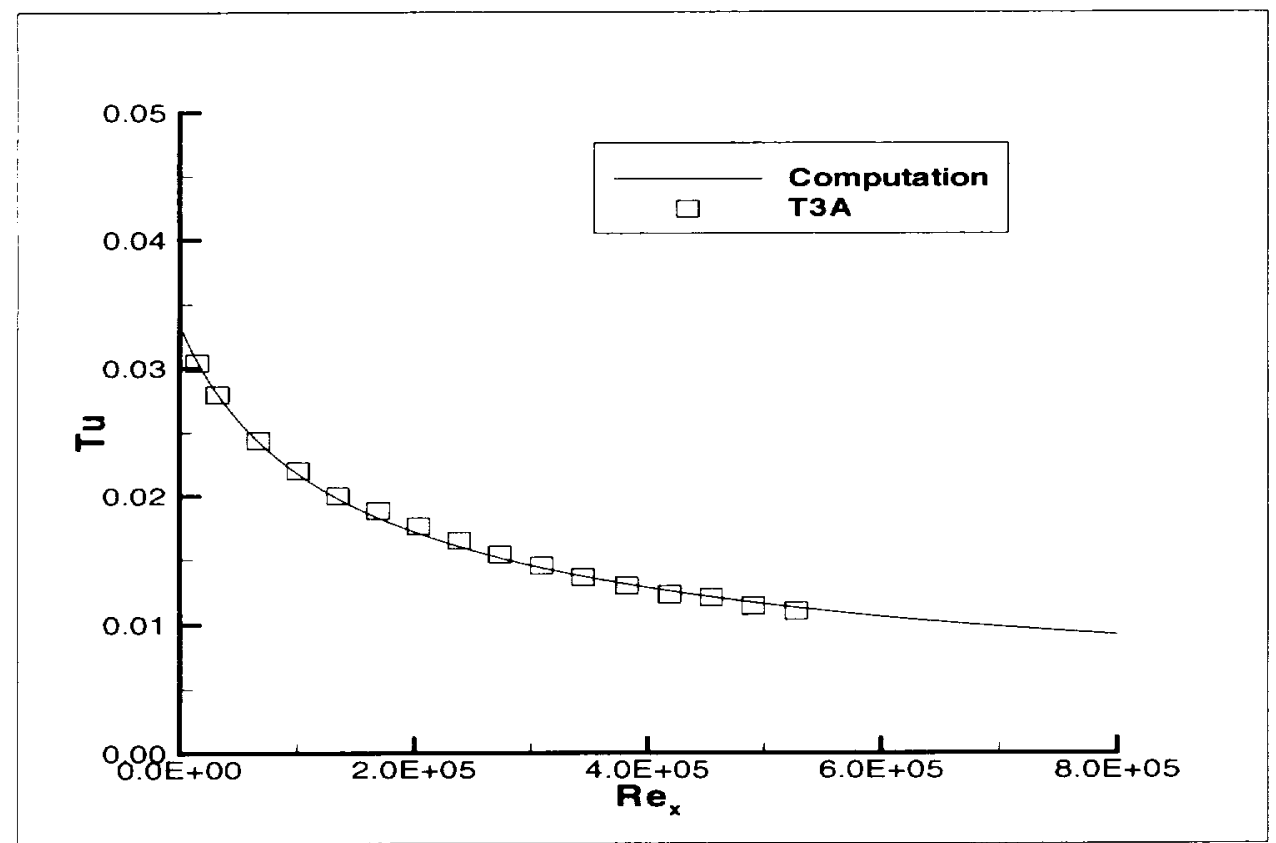

Figure 2: ('omparison of freestream turbulence intensity for T3A case. 


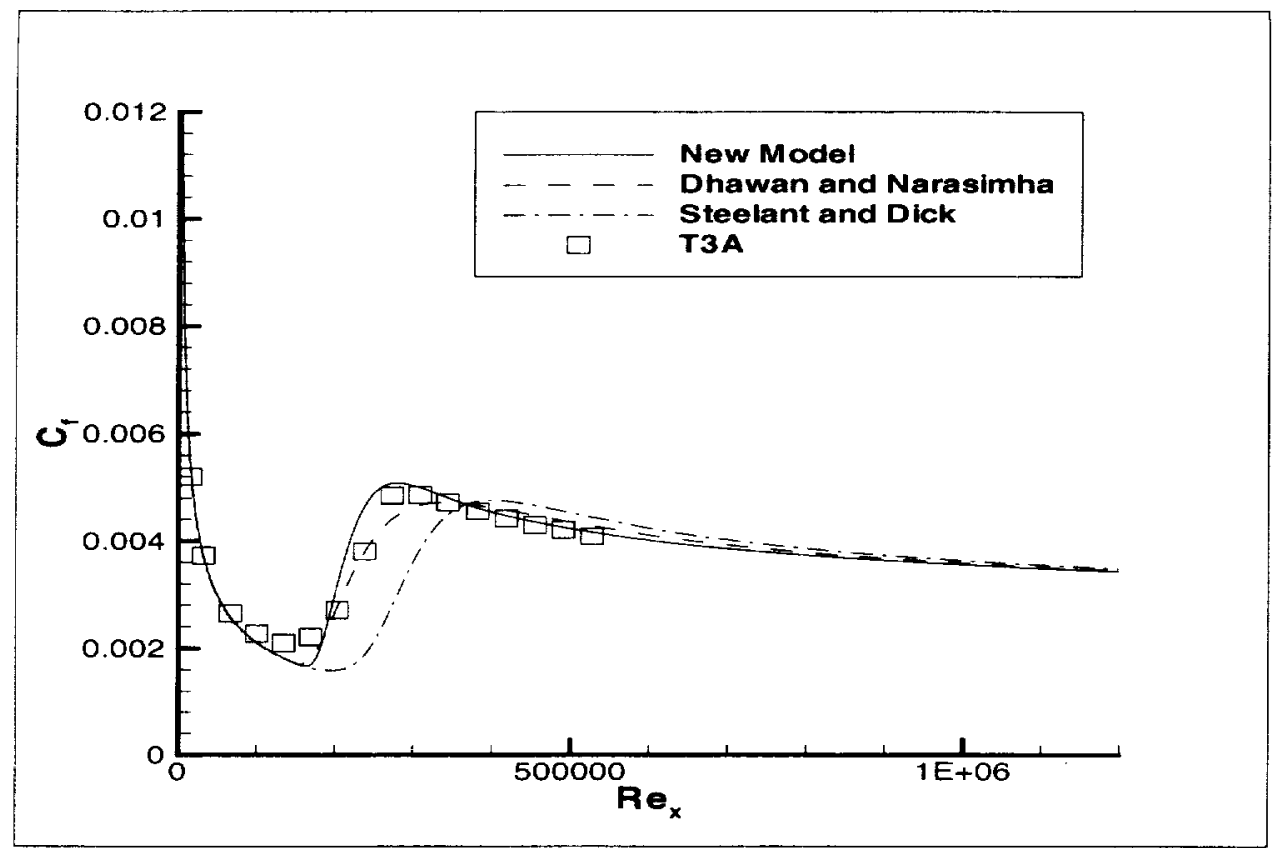

Figure 3: ('omparison of skin-friction coefficient for T3A case.

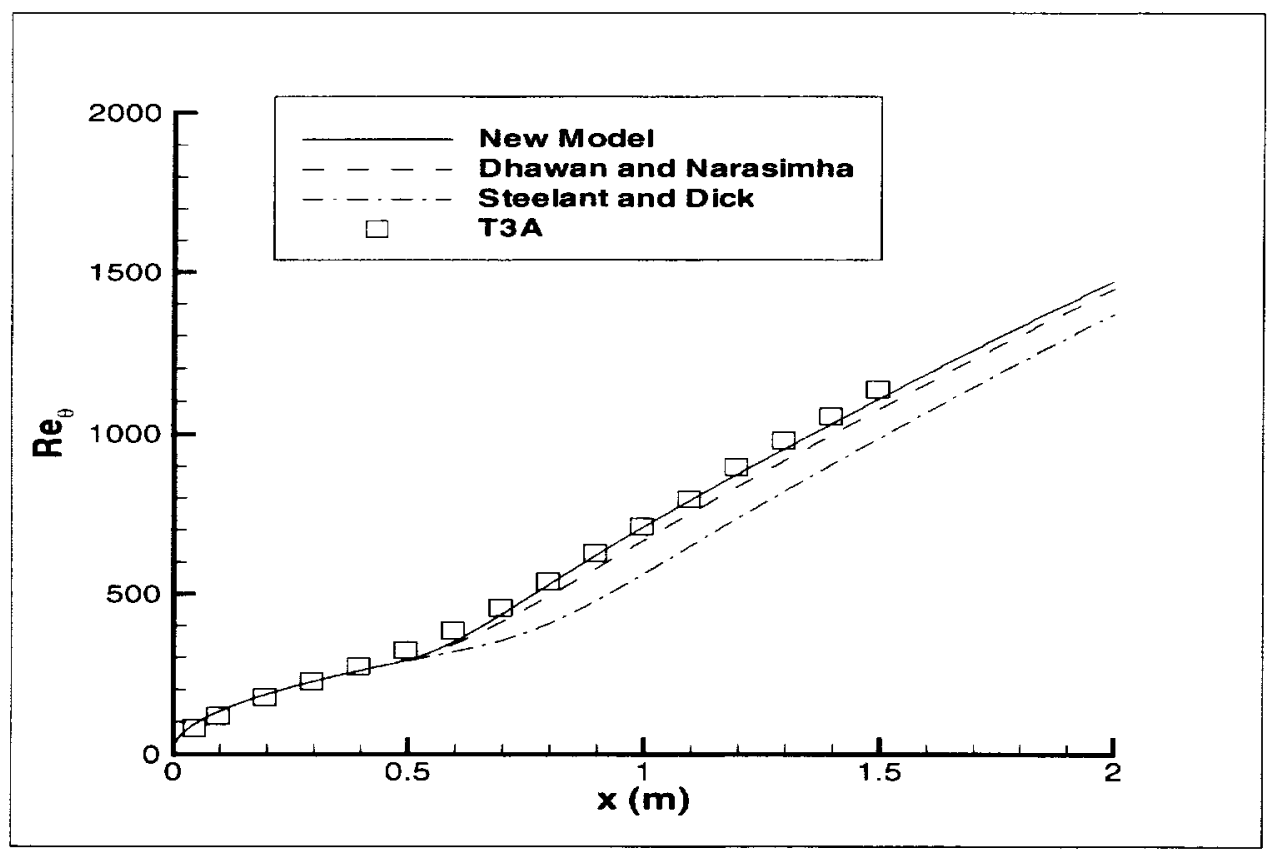

Figure 4: Comparison of Reynolds number based on momentum-thickness for T3A case. 


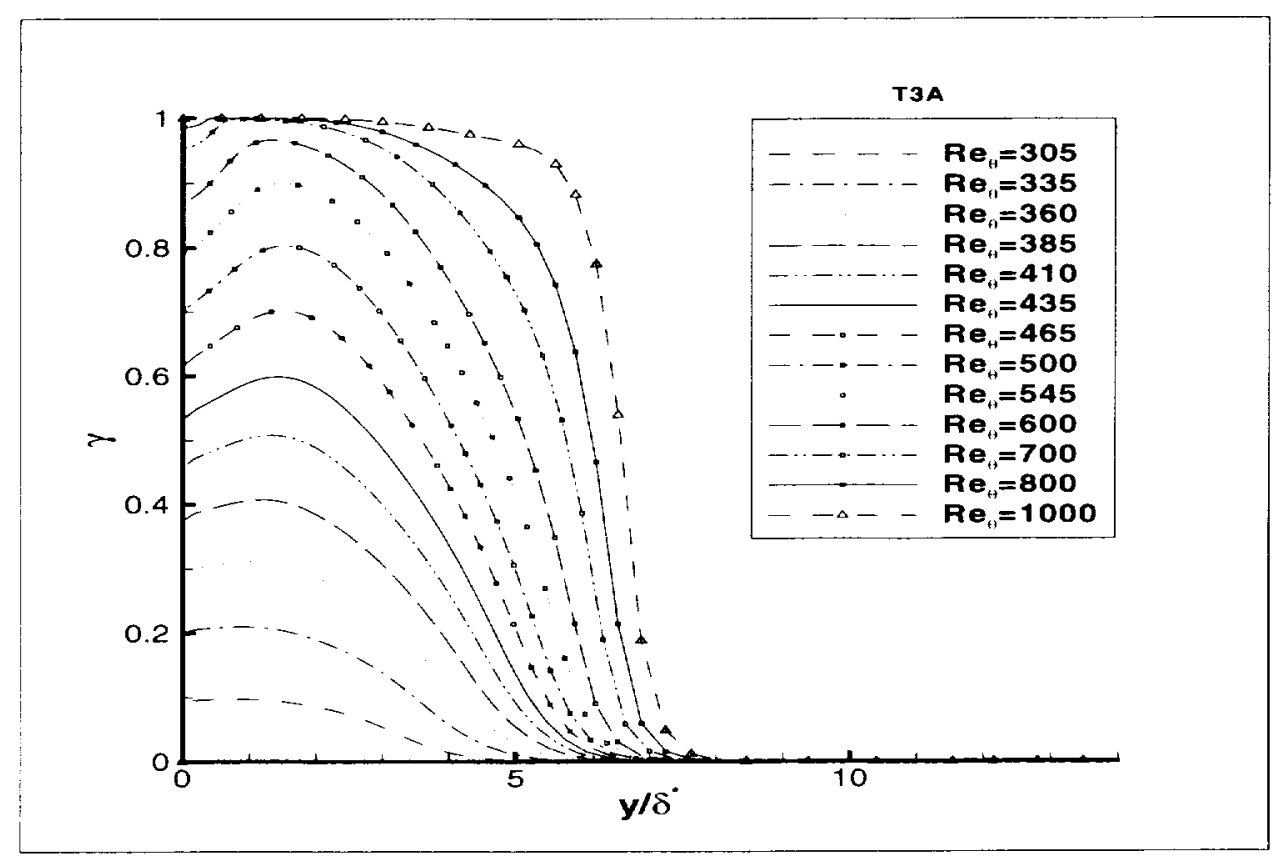

Figure 5: Intermittency factor profiles for T:3A case. 


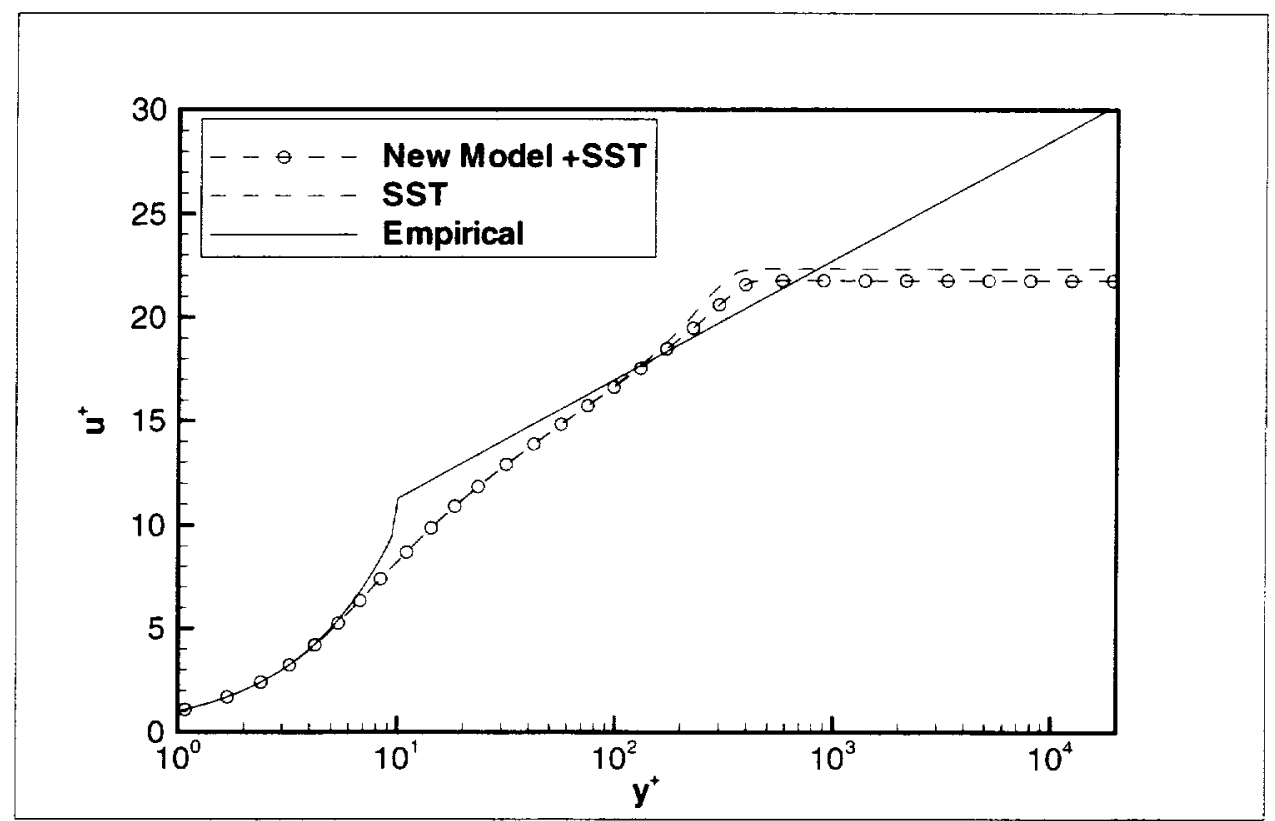

(a) Comparison of velocity profiles at $\mathrm{Re}_{\theta}=1000$.

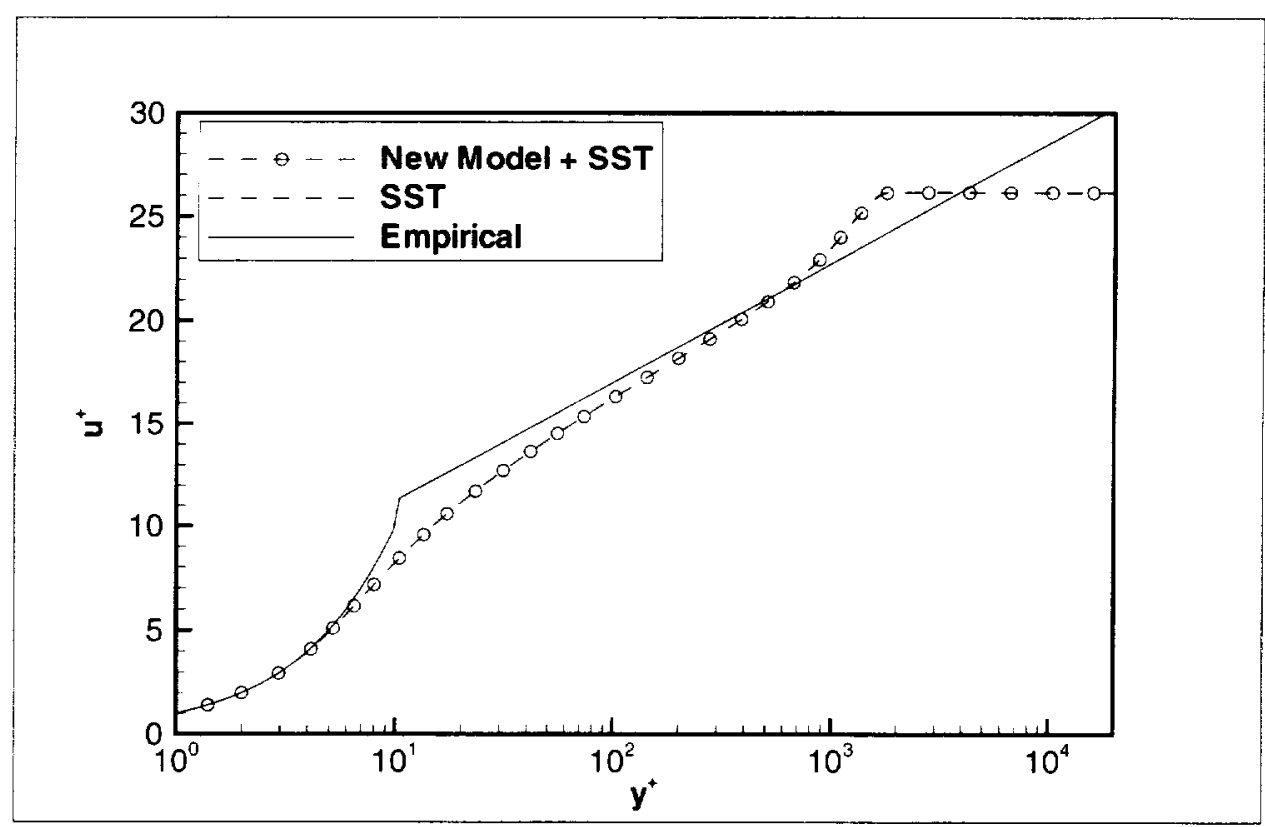

(b) Comparison of velocity profiles at $\mathrm{Re}_{\theta}=5000$.

Figure 6: Comparison of velocity profiles at two stations. 


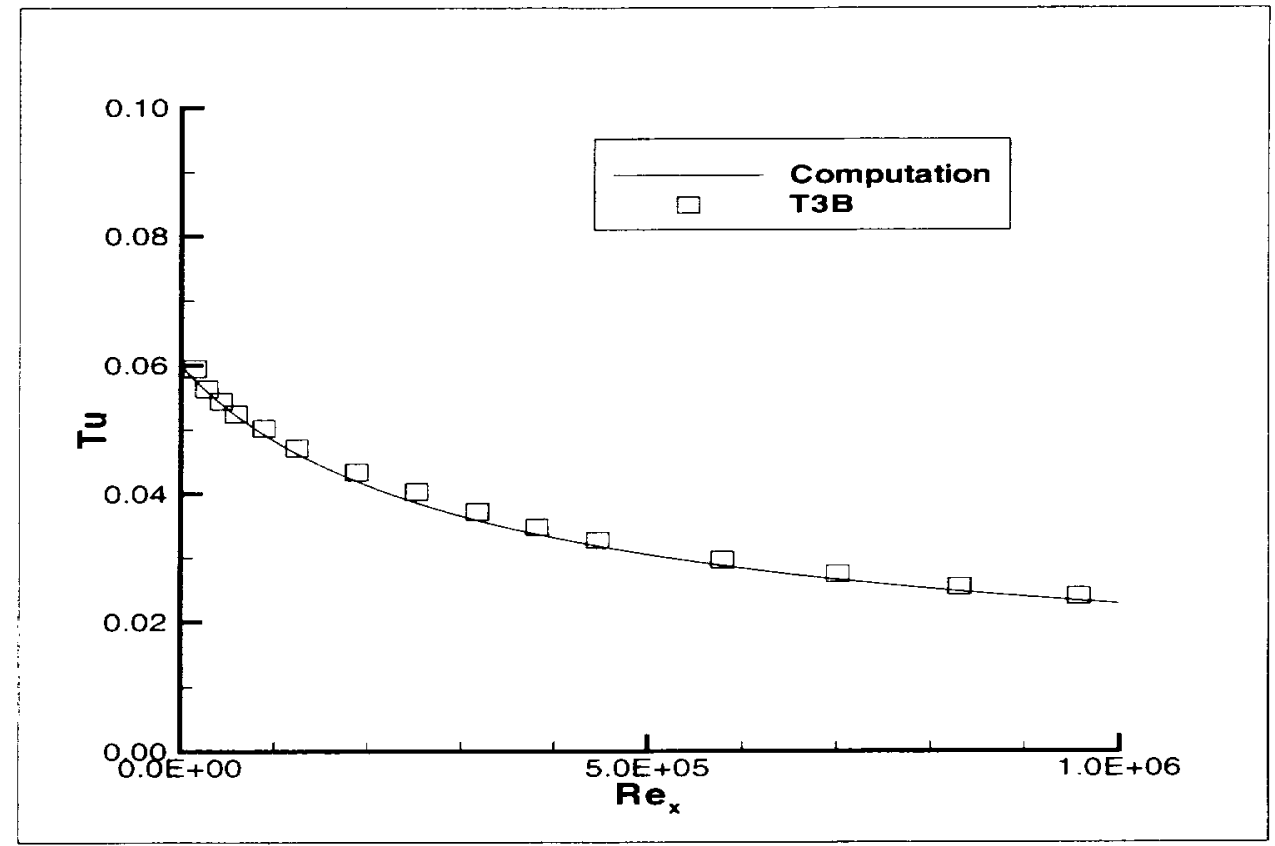

Figure 7: ('omparison of freestream turbulence intensity for T:3B case.

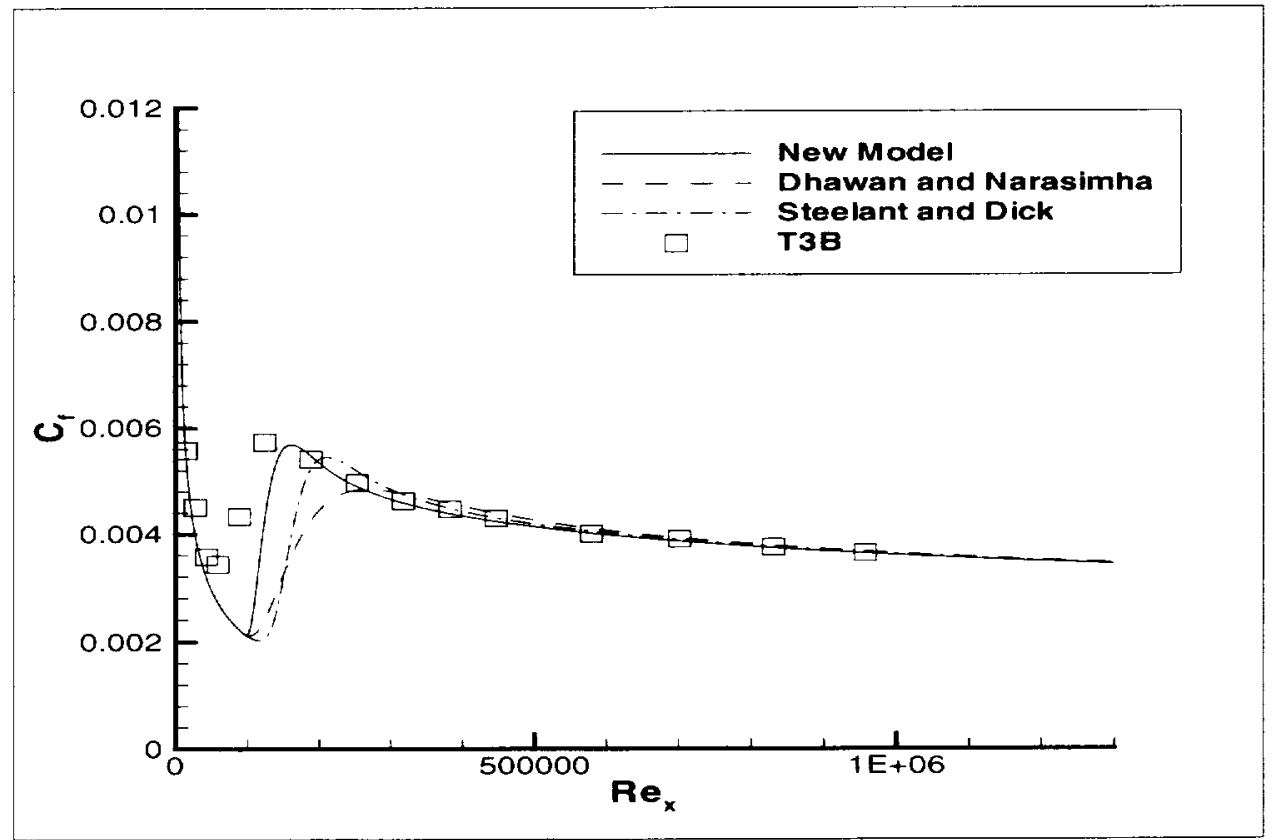

Figure s: Comparison of skin-friction coefficient for T:3B case. 


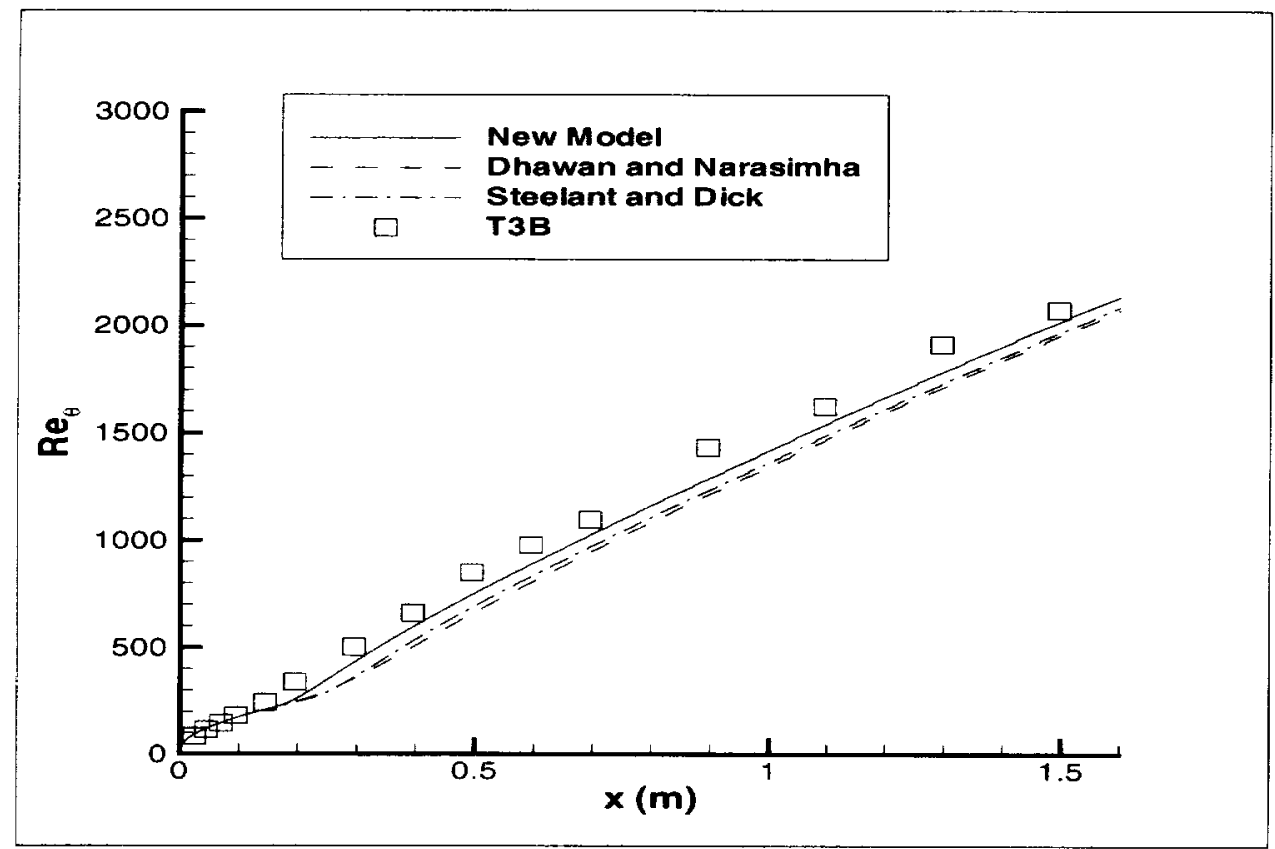

Figure 9: Comparison of Reynolds number based on momentum-thickness for T3B case.

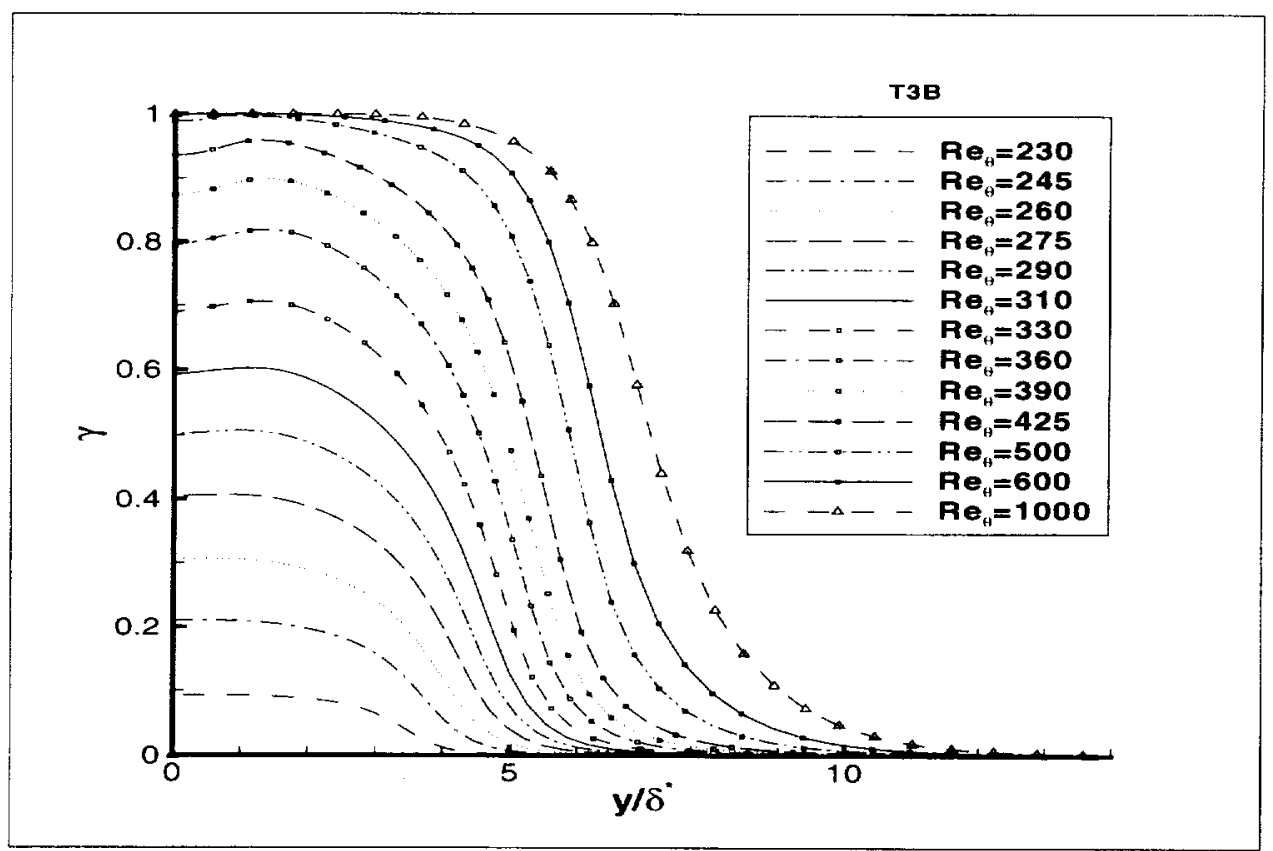

Figure 10: Intermittency factor profiles for T3B case. 


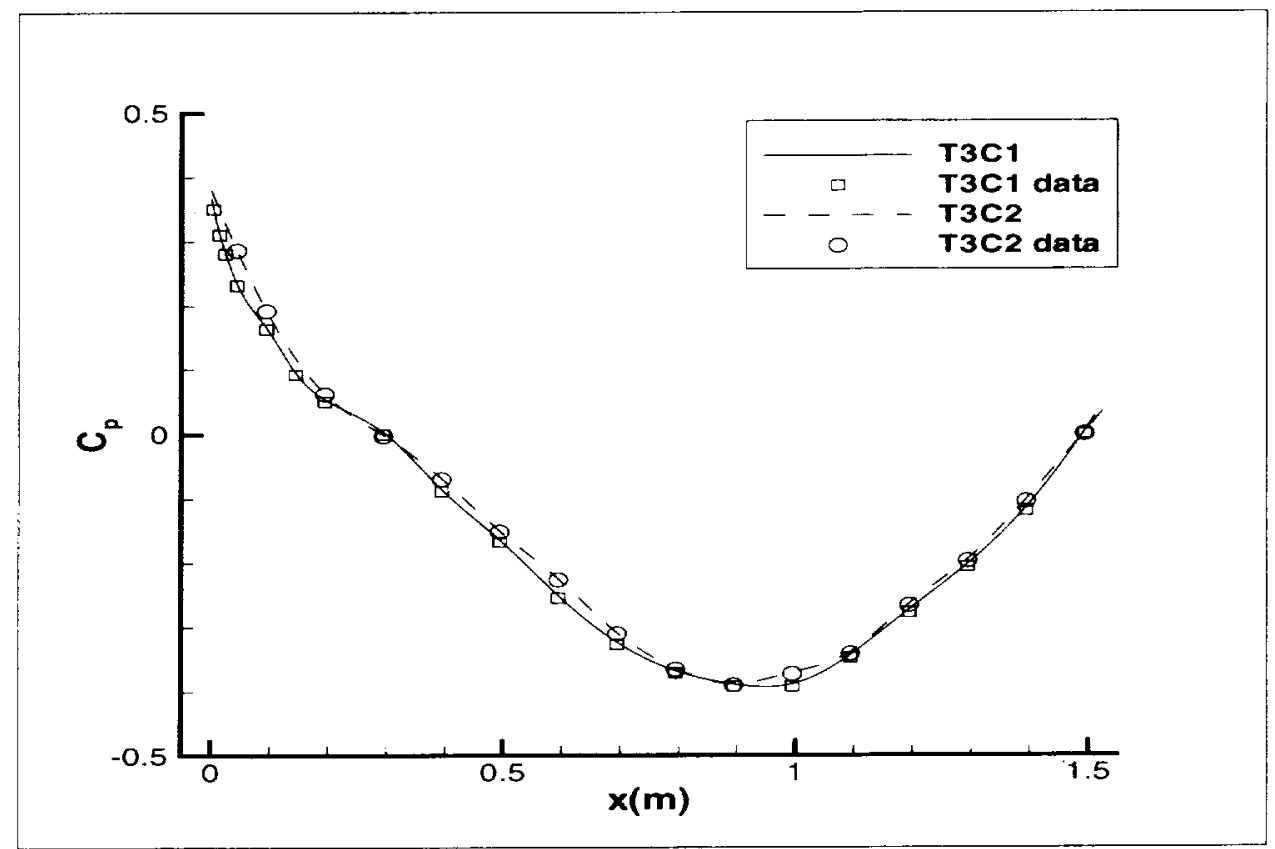

Figure 11: Pressure coefficient distributions for T:3('1 and T:3('2) cases.

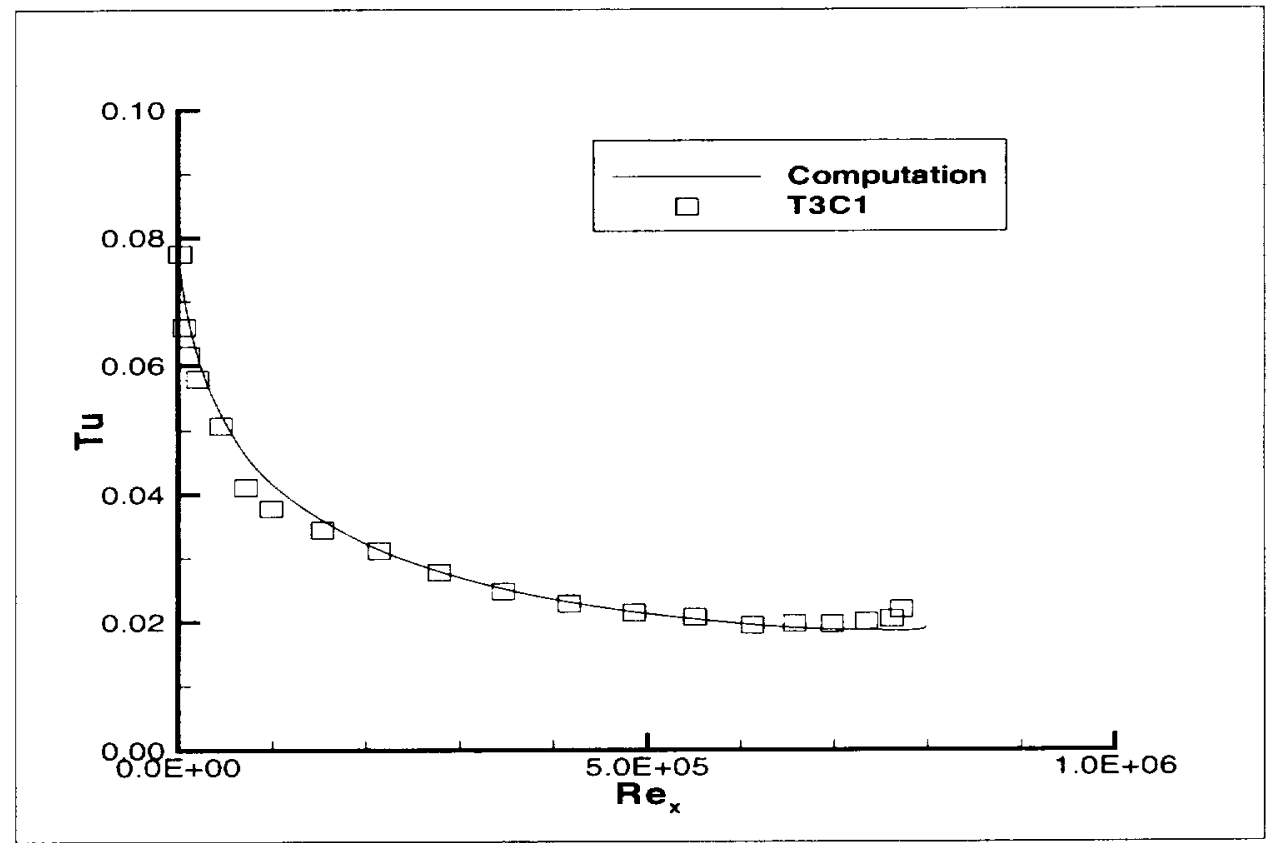

Figure 12: Comparison of freestream turbulence intensity for T'3('I case. 


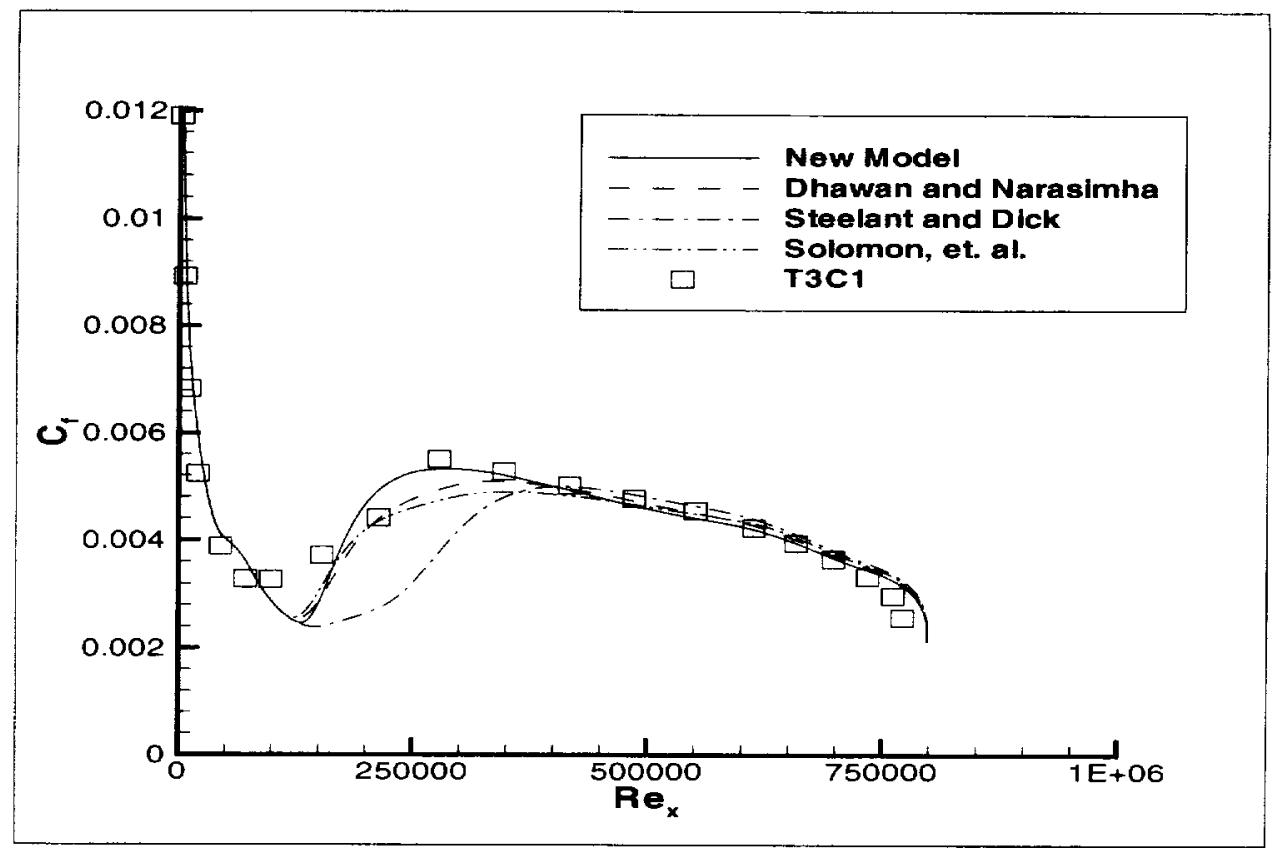

Figure 13: Comparison of skin-friction coefficient for T3C1 case.

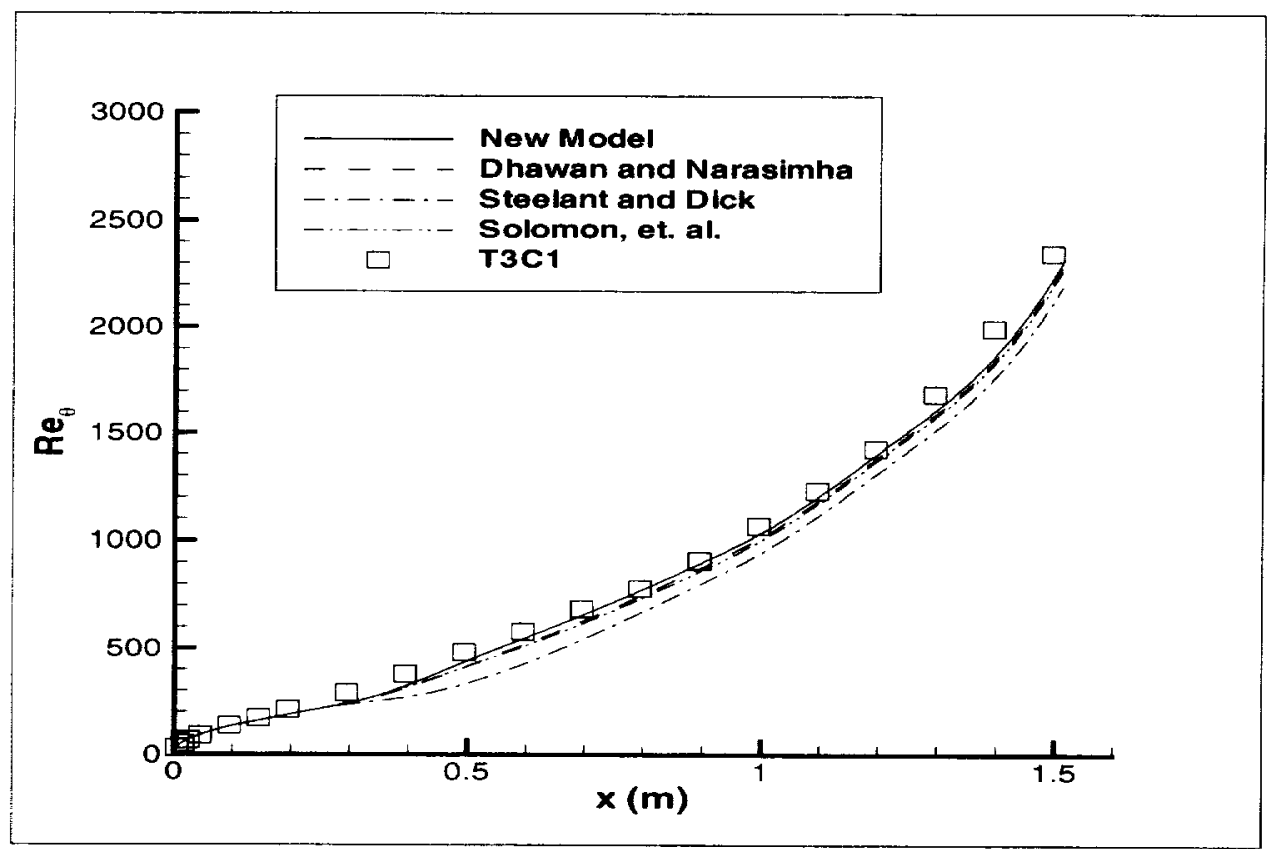

Figure 14: Comparison of Reynolds number based on momentum-thickness for T:3C'l case. 


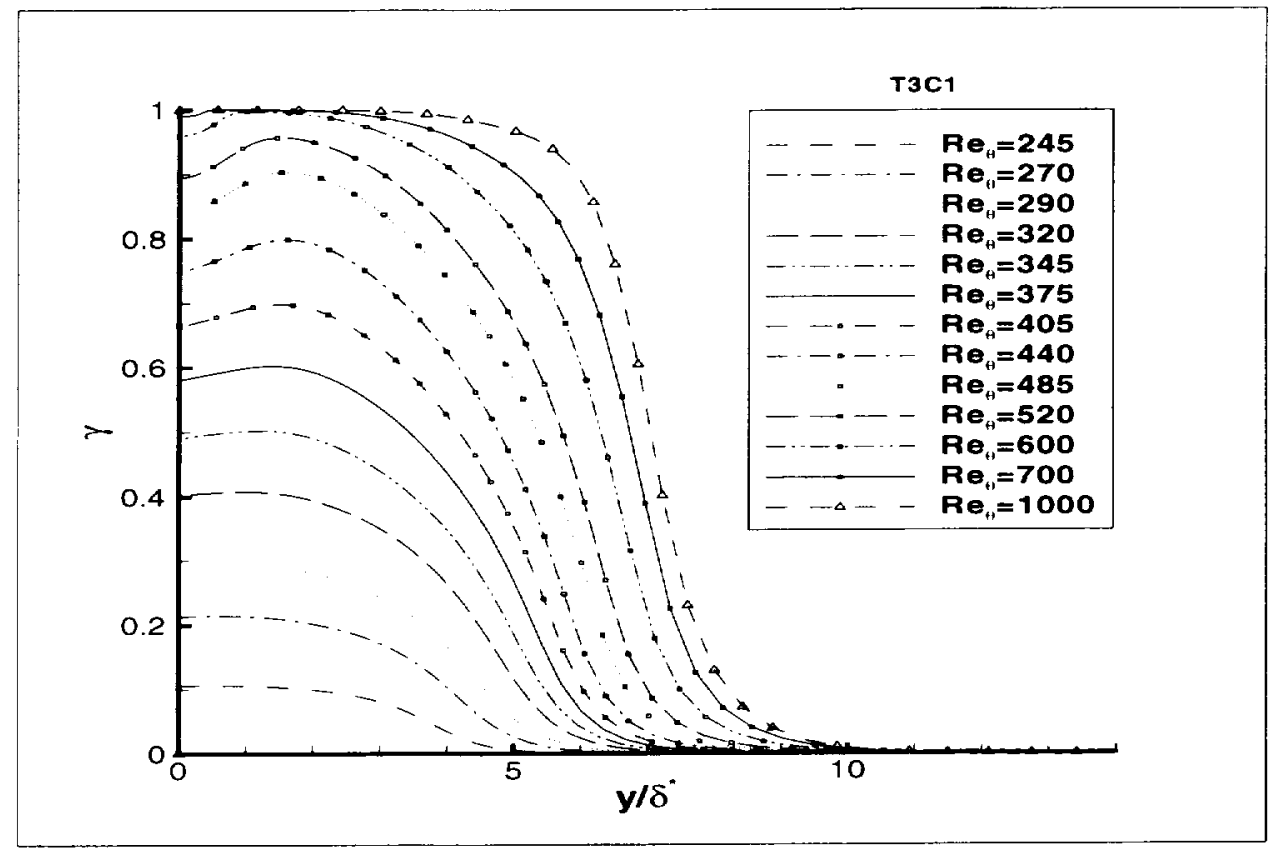

Figure 15: Intermittency factor profiles for T3('l case.

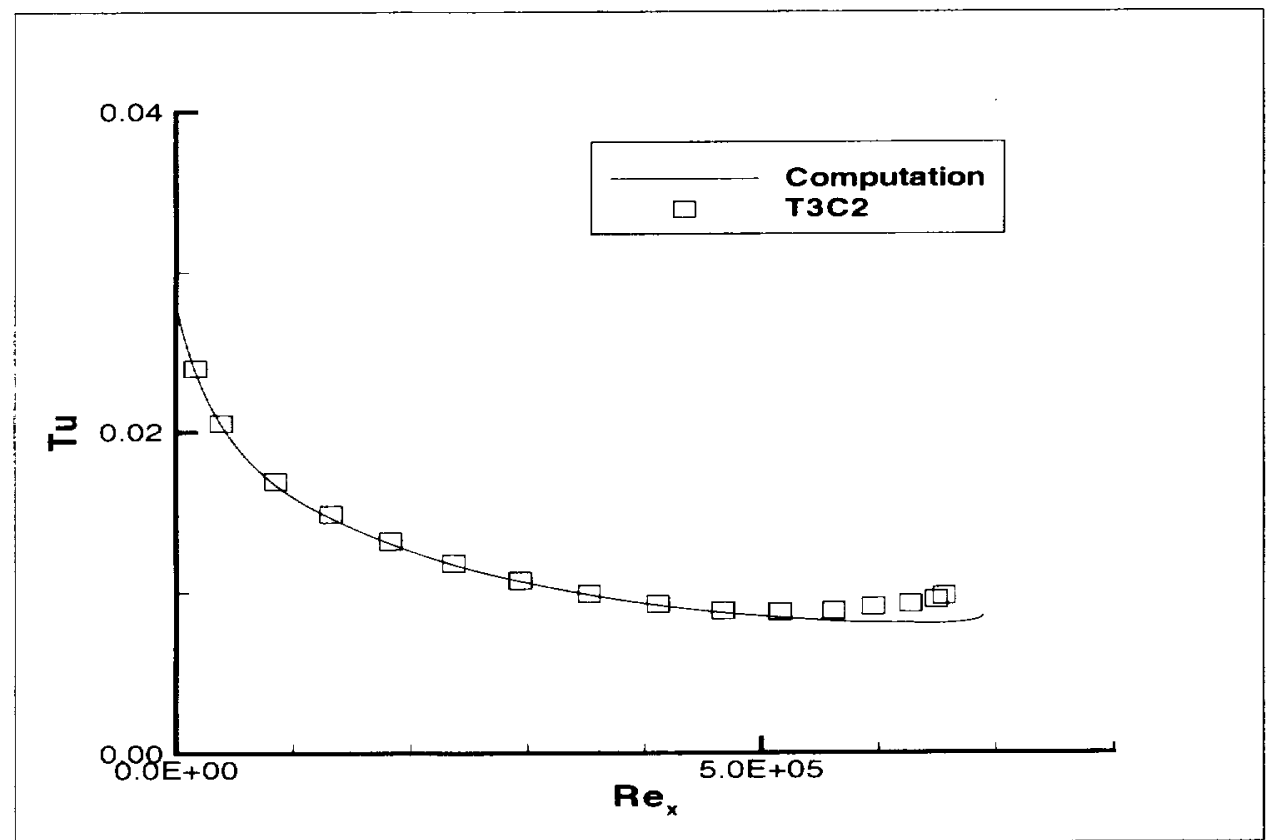

Figure 16: ('omparison of freestream turbulence intensity for T3( 2 case. 


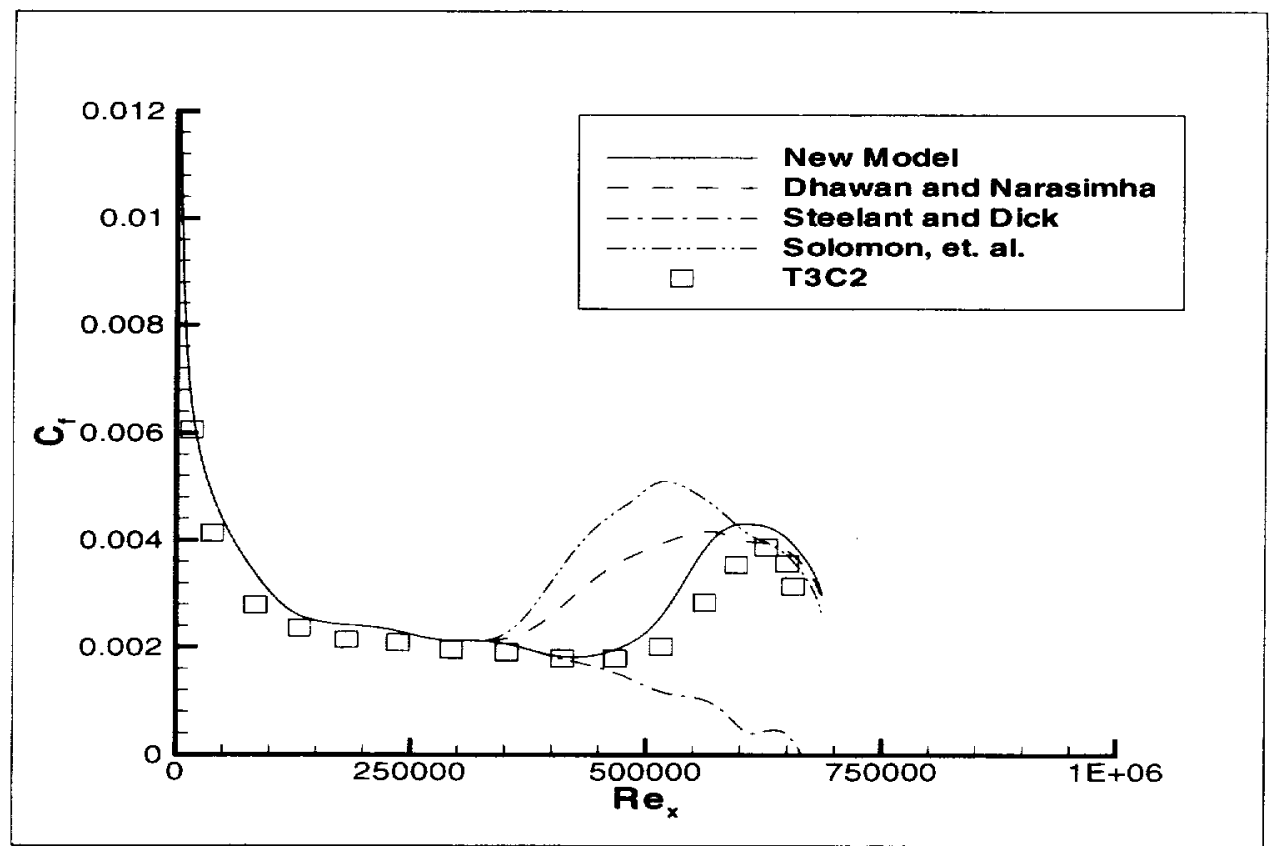

Figure 17: Comparison of skin-friction coefficient for $\mathrm{T} 3 \mathrm{C} 2$ case.

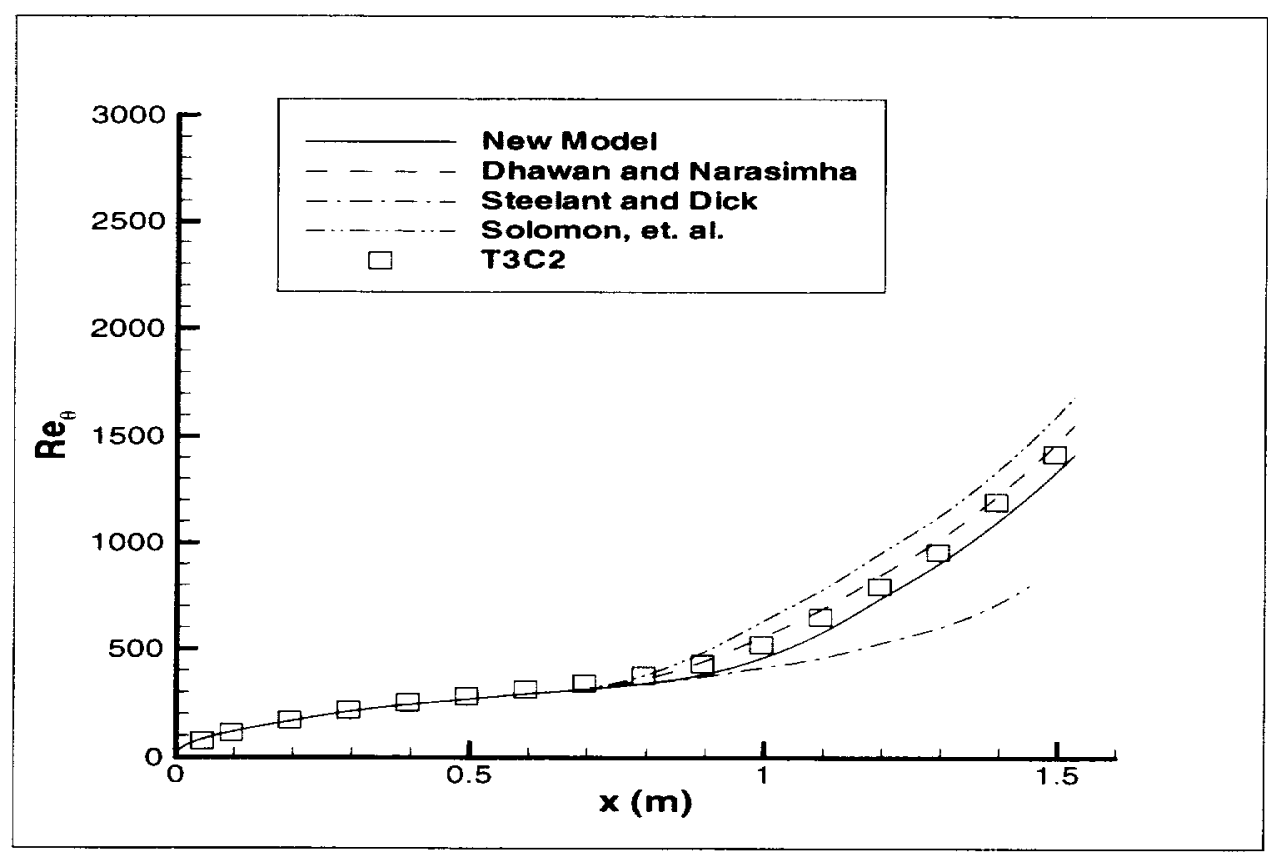

Figure 18: Comparison of Reynolds number based on momentum-thickness for T3C.2 case. 


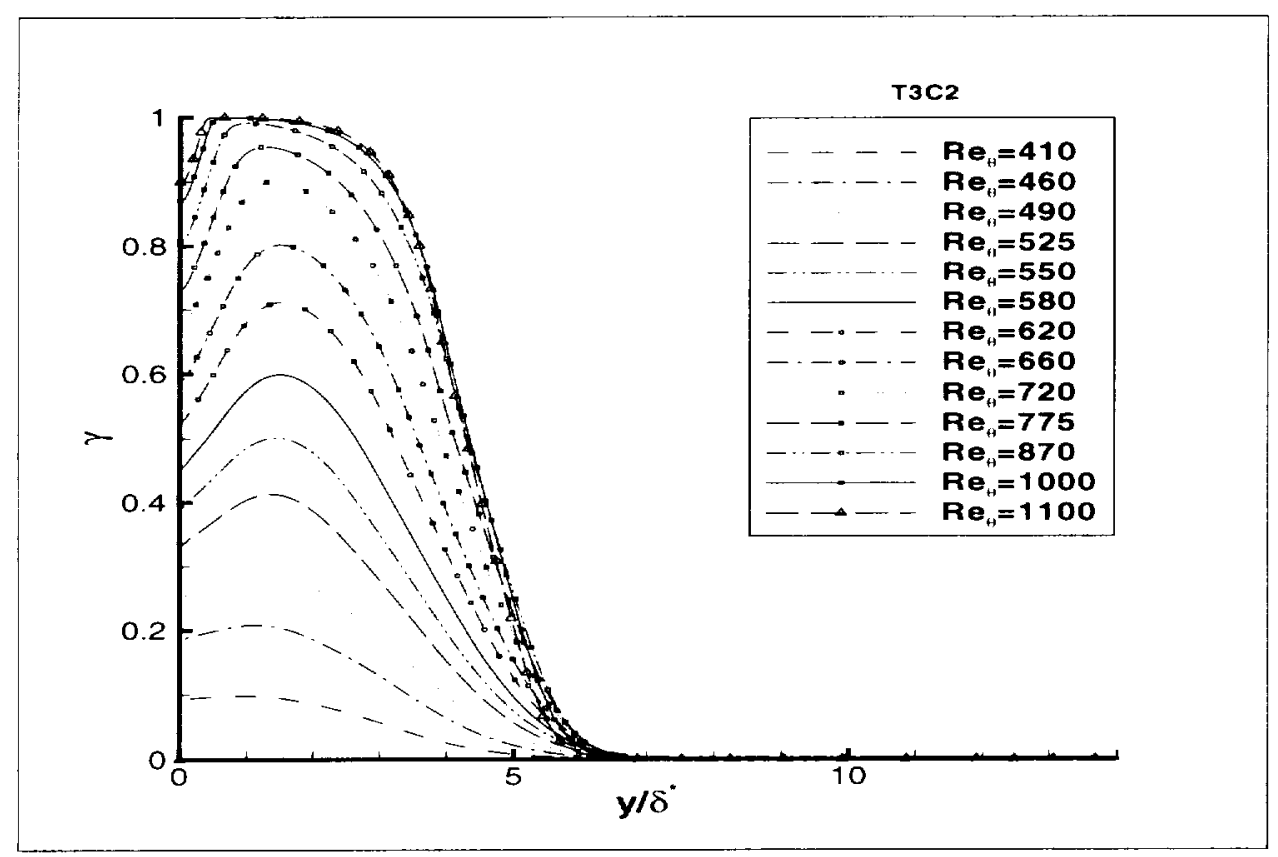

Figure 19: Internittency factor profiles for T:3('2 case. 


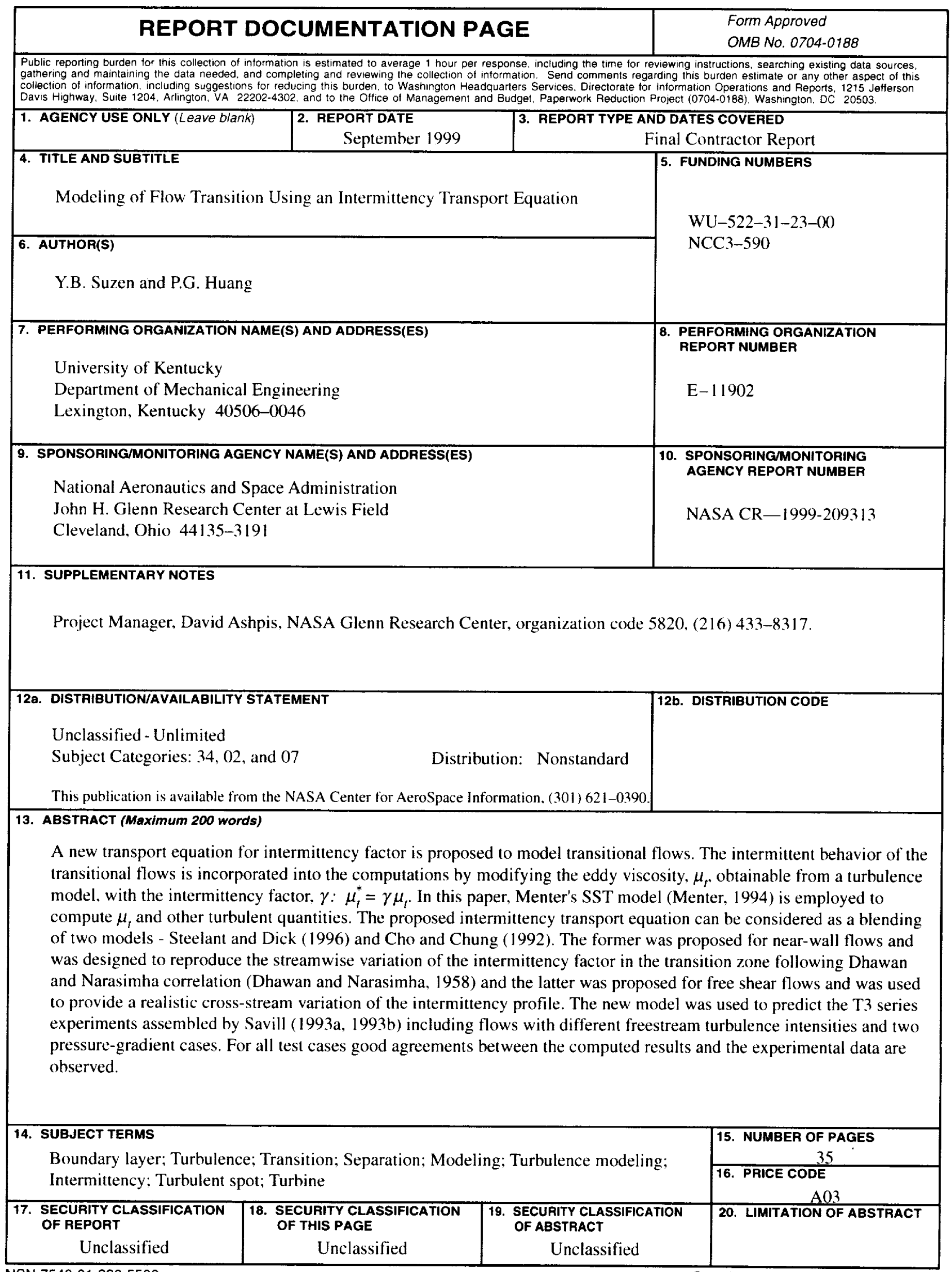



